Research article

urn:lsid:zoobank.org:pub:D5FDAA4D-D103-46E0-A65A-F0D27B77468F

\title{
Review of the Palaearctic species of Ismaridae Thomson, 1858 (Hymenoptera: Diaprioidea)
}

\author{
Chang-Jun KIM ${ }^{1}$, David G. NOTTON ${ }^{2}$, Frode ØDEGAARD ${ }^{3} \&$ Jong-Wook LEE ${ }^{4, *}$ \\ ${ }^{1}$ Division of Gardens and Education, Korea National Arboretum, Pocheon, 11186, Republic of Korea. \\ ${ }^{2}$ Department of Life Sciences, Insects Division, Darwin Centre - room 315, \\ The Natural History Museum, Cromwell Road, London, SW7 5BD, United Kingdom. \\ ${ }^{3}$ Norwegian Institute for Nature Research, Høgskoleringen 9, 7034 Trondheim, Norway. \\ ${ }^{4}$ Department of Life Sciences, Yeungnam University, Gyeongsan, 38541, Republic of Korea. \\ *Corresponding author: jwlee1@ynu.ac.kr \\ 1Email: hades821@gmail.com \\ ${ }^{2}$ Email: d.notton@nhm.ac.uk \\ ${ }^{3}$ Email: frode.odegaard@nina.no

\footnotetext{
${ }^{1}$ urn:Isid:zoobank.org:author:484B44D3-C017-4D3C-AEE6-9384A10A5915

${ }^{2}$ urn:1sid:zoobank.org:author:2E452EF2-703C-47C0-8432-6AB9C05AC06A

${ }^{3}$ urn:urn:1sid:zoobank.org:author:480435DB-5D54-458A-B0D8-01C59B057E99

${ }^{4}$ urn:1sid:zoobank.org:author:29B0EAD6-5F06-46DA-A384-69FDE8CBEF34
}

\begin{abstract}
The Palaearctic species of Ismaridae Thomson, 1858 are reviewed. Thirteen species of Ismaridae are recognized from the Palaearctic. Five species are described as new: Ismarus brevis Kim \& Lee sp. nov. from the Russian Far East and South Korea; I. distinctus Kim, Notton \& Ødegaard sp. nov. from Norway and the United Kingdom; I. excavatus Kim \& Lee sp. nov. from China, Japan and South Korea; I. similis Kim, Notton \& Lee sp. nov. from the United Kingdom and I. tripotini Kim \& Lee sp. nov. from South Korea. Ismarus apicalis Kolyada \& Chemyreva, 2016 is newly recorded from China, France, Japan and South Korea; I. dorsiger (Haliday, 1831) from France, Montenegro, Norway, South Korea and Switzerland; I. flavicornis (Thomson, 1858) from Bulgaria and Norway; I. grandis Alekseev, 1978, I. halidayi Förster, 1850 and I. multiporus Kolyada \& Chemyreva from Japan and South Korea; I. rugulosus Förster, 1850 from Austria and I. spinalis Kolyada \& Chemyreva, 2016 from China, Japan and South Korea. An identification key to all species found in the Palaearctic region is presented.
\end{abstract}

Keywords. Ismarus, review, new species, variation, new distribution records.

Kim C.-J., Notton D.G., Ødegaard F. \& Lee J.-W. 2018. Review of the Palaearctic species of Ismaridae Thomson, 1858 (Hymenoptera: Diaprioidea). European Journal of Taxonomy 417: 1-38.

https://doi.org/10.5852/ejt.2018.417 


\section{Introduction}

Ismaridae Thomson, 1858, a monotypic family of superfamily Diaprioidea Haliday, 1833 has been previously reported from all zoogeographical regions except the Ethiopian and Malagasy and 38 species have been described (Johnson 1992; Liu et al. 2011; Kolyada \& Chemyreva 2016; Comério et al. 2016; various contributors 2016). In addition, specimens are now known from the Ethiopian and Malagasy regions (authors' unpublished data). We have followed Sharkey et al. (2012) in using Ismaridae at family rank, a viewpoint which has been widely adopted by others (Comério 2014; Peeters 2015; Comério et al. 2016; Kim 2016; Kolyada \& Chemyreva 2016). In the century since the studies of Kieffer $(1908,1916)$ on this family, several regional revisions have been made: Britain (Nixon 1957); former Czechoslovakia (Masner 1957); Finland (Hellén 1964); the New World (Masner 1976); Russia (Kozlov 1978; Kolyada \& Chemyreva 2016) and China (Liu et al. 2011).

Members of Ismaridae prefer to inhabit wooded areas at higher elevations in warmer climatic zones and at low elevations in cooler climatic zones. They are relatively rarely collected, but at least some of the Palaearctic species are very common (e.g., Ismarus halidayi Förster, 1850). Available host data show that species of Ismaridae are hyperparasitoids of planthoppers (Hemiptera Linnaeus, 1758, Auchenorrhyncha Duméril, 1806) via Dryinidae Haliday, 1833 (Hymenoptera Linnaeus, 1758: Chrysidoidea Latreille, 1802) (Chambers 1955, 1981; Nixon 1957; Wall 1967; Kozlov 1971; Masner 1976; Jervis 1979; Tussac \& Tussac 1991; Olmi 2000). Dryinidae are sometimes very effective natural enemies, because they are parasitoids and often also predators of Auchenorrhyncha including leafhoppers, planthoppers and treehoppers that are important pests of cultivated and forest plants (Guglielmino \& Olmi 1997, 2006, 2007; Olmi 1984, 1999a, 2000; Guglielmino et al. 2013). The importance of Dryinidae has been widely recognized, resulting in some biological control programmes (Swezey 1928; Williams 1931; Dumbleton 1937; Charles 1989; Girolami \& Camporese 1994; Olmi 1999b; Mora-Kepfer \& Espinoza 2009). Since Ismaridae can be parasitoids of beneficial dryinids, so Ismaridae may potentially be detrimental for biological control.

Recently, we have accumulated many specimens of Ismaridae during surveys of Palaearctic Diaprioidea. Among them, thirteen species of Ismaridae are recognized here, of which five are considered to be new to science (Table 1). All the Palaearctic species are described and keyed.

\section{Material and methods}

The terminology used in the present study follows that of Masner (1976) and Masner \& García (2002). The images were captured with an Axiocam HRc camera through a Discovery V20 stereo microscope (Carl Zeiss, Oberkochen, Germany) and were produced with AxioVision40AC software (Carl Zeiss, Oberkochen, Germany) or with a Leica DFC 495 camera through a Leica M205A Stereozoom stereo microscope (Leica, Microsystems, Solms, Germany) and were produced with LAS software (v. 4.1.0, Leica Microsystems, Switzerland). Final plates were prepared in Adobe Photoshop CS6 (Adobe Systems Incorporated, San Jose, United States of America). Measurements were generated using LAS software or micrometer eyepiece.

The following abbreviations are used throughout the text:

$\mathrm{LOL}=$ distance between the inner edges of lateral ocellus and median ocellus

$\mathrm{OOL}=$ distance from the outer edge of lateral ocellus to the compound eye

POL $=$ distance between the inner edges of the two lateral ocelli

MT = Malaise trap

YPT = yellow pan trap 
Table 1. An overview of Palaearctic species of Ismarus Haliday, 1835. Abbreviations: E.Pal = Eastern Palaearctic; W.Pal = Western Palaearctic; * = new country record; $\mathrm{O}=$ known; $\mathrm{X}=$ unknown.

\begin{tabular}{|c|c|c|c|c|}
\hline Species & $\begin{array}{l}\text { Se } \\
+9 \\
\end{array}$ & & Region & Distribution \\
\hline I. apicalis Kolyada \& Chemyreva, 2016 & $\mathrm{O}$ & $\mathrm{O}$ & E.Pal, W.Pal & $\begin{array}{c}{ }^{*} \text { China, }{ }^{*} \text { France, *Japan, *South Korea, } \\
\text { Russia (Far East) }\end{array}$ \\
\hline I. brevis Kim \& Lee sp. nov. & $\mathrm{X}$ & $\mathrm{O}$ & E.Pal & Russia (Far East), South Korea \\
\hline I. distinctus Kim, Notton \& Ødegaard sp. nov. & $\mathrm{O}$ & $\mathrm{O}$ & W.Pal & Norway, United Kingdom \\
\hline I. dorsiger (Haliday, 1831) & $\mathrm{O}$ & $\mathrm{O}$ & $\begin{array}{l}\text { E.Pal, W.Pal, } \\
\text { Oriental }\end{array}$ & $\begin{array}{c}\text { *South Korea, Europe (*France, } \\
\text { *Montenegro, *Norway, *Switzerland), } \\
\text { China (Yunnan) }\end{array}$ \\
\hline I. excavatus Kim \& Lee sp. nov. & $\mathrm{O}$ & $\mathrm{O}$ & E.Pal & China, Japan, South Korea \\
\hline I. flavicornis (Thomson, 1858) & $\mathrm{O}$ & $\mathrm{O}$ & Holarctic & $\begin{array}{c}\text { Europe (*Bulgaria, }{ }^{*} \text { Norway), Canada, } \\
\text { USA }\end{array}$ \\
\hline I. grandis Alekseev, 1978 & $\mathrm{O}$ & $\mathrm{O}$ & E.Pal & *Japan, *South Korea, Russia (Far East) \\
\hline I. halidayi Förster, 1850 & $\mathrm{O}$ & $\mathrm{O}$ & Holarctic & $\begin{array}{c}\text { *Japan, *South Korea, Canada, China, } \\
\text { Mongolia, Europe }\end{array}$ \\
\hline I. multiporus Kolyada \& Chemyreva, 2016 & $\mathrm{O}$ & $\mathrm{O}$ & E.Pal & *Japan, *South Korea, Russia (Far East) \\
\hline I. rugulosus Förster, 1850 & $\mathrm{O}$ & $\mathrm{O}$ & Holarctic & Europe (*Austria), Canada, USA \\
\hline I. similis Kim, Notton \& Lee sp. nov. & $\mathrm{O}$ & $\mathrm{X}$ & W.Pal & United Kingdom \\
\hline I. spinalis Kolyada \& Chemyreva, 2016 & $\mathrm{O}$ & $\mathrm{O}$ & E.Pal, W.Pal & $\begin{array}{c}\text { *China, *Japan, *South Korea, } \\
\text { Kazakhstan, Russia (European, Far East) }\end{array}$ \\
\hline I. tripotini sp. nov. Kim \& Lee & $\mathrm{O}$ & $\mathrm{X}$ & E.Pal & South Korea \\
\hline
\end{tabular}

The identification of species was confirmed by reference to type material where this was available; in these cases, type specimen data is cited. Where type specimens were not available, identifications were made by reference to Nixon (1957), Masner (1976), Kolyada \& Chemyreva (2016), Kolyada (pers. comm.) and Liu et al. (2011), and previously identified specimens in the collections of NHMUK, CNC and YNU.

In this study, the first author examined a large amount of unidentified Palaearctic Ismaridae provided by Lubomír Masner (CNCI), David Notton (NHMUK), Frode Ødegaard (NINA) and Jong-Wook Lee (YNU). Material in the NHMUK was identified by DGN, in the NINA was identified by FØ and all material was identified or confirmed by CJK.

Collection abbreviations:

$\mathrm{CNCI}=$ Canadian National Insect Collection, Ottawa, Canada

DNPC = David G. Notton Personal Collection

HNHM = Hungarian Natural History Museum, Budapest, Hungary

KNA $=$ Korea National Arboretum, Pocheon, South Korea

NHMUK $=$ Natural History Museum, London, United Kingdom

NHRS $=$ Swedish Museum of Natural History, Stockholm, Sweden

NINA $=$ Norwegian Institute for Nature Research, Trondheim, Norway

YNU = Yeungnam University, Gyeongsan, South Korea 


\section{Results}

Class Hexapoda Blainville, 1816

Order Hymenoptera Linnaeus, 1758

Superfamily Diaprioidea Haliday, 1833

Family Ismaridae Thomson, 1858

\section{Diagnosis}

This family can be distinguished from other families in this superfamily by the following combination of characters: low insertion of antennae; antennal shelf not developed; antenna of female 15-segmented and male 14-segmented; notauli are largely absent, but represented anteriorly by pits; mesoscutum strongly arched in lateral view; base of second tergite with median furrow.

\section{Identification key to Palaearctic species of Ismarus Haliday, 1835}

\section{Females}

The female of $I$. brevis sp. nov. is unknown.

1. Body mainly pale yellowish to yellow, except at least mesoscutum, scutellum black (Fig. 1B) ...... Ismarus dorsiger (Haliday, 1831)

- Body mainly dark brown or black (Fig. 1A, C-G) . .2

2. Posterior part of scutellum finely coriaceous or rugose (Fig. 4D-E) ...........................

- Posterior part of scutellum smooth, sculptureless (Figs 5D, 6C, 7D) ..........................

3. Antenna uniformly brown to dark brown (Fig. 1E); posterior part of scutellum coriaceous; mesopleuron coriaceous-rugulose; metasoma deeply scaly-reticulate ....

Ismarus rugulosus Förster, 1850

- Antenna uniformly bright yellow except for apical segment brownish (Fig. 4A, D); posterior part of scutellum rugose (Fig. 4D-E); mesopleuron smooth (Fig. 4A); metasoma weakly rugulose (Fig. 4A, D) .....................................Ismarus distinctus Kim, Notton \& Ødegaard sp. nov.

4. Scutellum truncate posteriorly, with hind margin straight (Fig. 7D); hind tibia abruptly swollen (Fig. 7B) .Ismarus tripotini Kim \& Lee sp. nov.

- Scutellum rounded posteriorly (Figs 5D, 6C); hind tibia gradually swollen (Fig. 5B, 6A) ..........5

5. Mesopleuron with a continuous zone of sculpture extending from its anteroventral corner up to meso-metapleural suture (Figs 1I, 6D)

- Mesopleuron without a continuous zone of sculpture (Figs 1H, 5B) .........................

6. Antenna uniformly yellow (Fig. 1G); mesopleuron with deep longitudinal wrinkles (Fig. 1I); base of second tergite with long median furrow, extending to $2 / 3$ of segment

Ismarus flavicornis (Thomson, 1858)

- Antenna not uniformly yellow (Fig. 6A); mesopleuron with deep punctures to short irregular wrinkles (Fig. 6D); base of second tergite with short median furrow, extending $0.4-0.5 \times$ length of tergite (Fig. 6E) Ismarus similis Kim, Notton \& Lee sp. nov.

7. Notauli with 5-8 pits; posterior half of S6 yellow ......Ismarus multiporus Kolyada \& Chemyreva

- Notauli with 1-2 pits; only margin of S6 yellow ......................................... 8

8. Antenna uniformly bright yellowish or only A15 brown (Fig. 1A, F) .......................

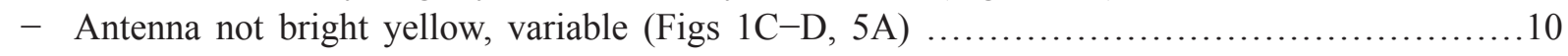


9. Antenna uniformly bright yellowish (Fig. 1F); anterior scutellar pit with median keel; radial cell as long as length of marginal vein (Fig. 1F); A7-A14 subquadrate

Ismarus spinalis Kolyada \& Chemyreva, 2016

- Antenna bright yellow, except A15 brown (Fig. 1A); anterior scutellar pit without median keel; radial cell $0.8 \times$ length of marginal vein (Fig. 1A); A7-A14 elongate

Ismarus apicalis Kolyada \& Chemyreva, 2016

10. POL much longer than OOL (Fig. 5C)

Ismarus excavatus Kim \& Lee sp. nov.

- POL slightly longer or as long as OOL

11. A4 as long as A1, slightly shorter than A3 (Fig. 1D)

Ismarus grandis Alekseev, 1978

- A4 shorter than A1 and A3 (Fig. 1C) Ismarus halidayi Förster, 1850

Males

The males of I. similis sp. nov. and I. tripotini sp. nov. are unknown.

1. A3 and A4 with keels (Fig. 2H) ..................Ismarus spinalis Kolyada \& Chemyreva, 2016

- A3 without keel, keel on A4 extending at least to $2 / 3$ of segment (Figs $3 \mathrm{~A}, 4 \mathrm{~B}, 5 \mathrm{E}$ ) ...............

2. Posterior part of scutellum finely coriaceous or punctate-rugose .............................. 3

- Posterior part of scutellum smooth, sculptureless (Figs 3D, 6C) ...............................4

3. Posterior part of scutellum coriaceous; mesopleuron coriaceous-rugulose; metasoma deeply scaly-reticulate

Ismarus rugulosus Förster, 1850

- Posterior part of scutellum punctate-rugose (Fig. 4D-E); mesopleuron smooth (Fig. 4A); metasoma weakly rugulose (Fig. 4D-E) ......Ismarus distinctus Kim, Notton \& Ødegaard sp. nov.

4. Mandibles white; notauli absent ...........................Ismarus dorsiger (Haliday, 1831)

- Mandibles black; notauli present 5

5. Mesopleuron with a continuous zone of sculpture extending from its anteroventral corner up to meso-metapleural suture (Fig. 2C) Ismarus flavicornis (Thomson, 1858)

- Mesopleuron without a continuous zone of sculpture (Figs 2A, D-F, 3B, 5F) ..................6

6. Radial cell shorter than marginal vein (Fig. 2A) ......Ismarus apicalis Kolyada \& Chemyreva, 2016

- Radial cell as long as marginal vein (Figs 2D-F, 3B, 5F) ................................

7. Notauli with $5-8$ pits

Ismarus multiporus Kolyada \& Chemyreva, 2016

- Notauli with 1-2 pits (Figs 3D) ..............................................................

8. A3 shorter than A4 (Fig. 5F) ...........................................................

- A3 as long as or slightly longer than A4 (Fig. 3A) ....................................... 10

9. POL as long as OOL; antennal segments distinctly elongate, at least $2.0 \times$ width of each segment (Fig. 2D); A4 as long as A1 (Fig. 2D); A4 slightly excavate (Fig. 2D); base of second tergite with long median furrow, at least to $2 / 3$ of segment .............Ismarus grandis Alekseev, 1978

- POL longer than OOL; antennal segments not distinctly elongate, A5-A13 only slightly longer than wide (Fig. 2E); A4 shorter than A1 (Fig. 5F); A4 distinctly excavate (Fig. 5F); base of second tergite with short median furrow, extending $0.4 \times$ length of tergite ... Ismarus excavatus Kim \& Lee sp. nov.

10. POL longer than OOL (Fig. 3C); A7-A13 quadrate, as long as wide each segment (Fig. 3A); notauli present anteriorly as large pits (Fig. 3D)

Ismarus brevis Kim \& Lee sp. nov.

- POL as long as OOL; A7-A13 longer than wide each segment (Fig. 2E); notauli present anteriorly, as oblique, elongate pits

Ismarus halidayi Förster, 1850 
Ismarus apicalis Kolyada \& Chemyreva, 2016

Figs 1A, 2A

Ismarus apicalis Kolyada \& Chemyreva, 2016: 5.

\section{Diagnosis}

Antenna yellow with female A15 and male apical segments one to four darkened; A7-A14 elongate in both sexes; male A3 without keel, keel on A4 extending 0.85-0.95 of segment; radial cell slightly shorter than length of marginal vein in both sexes.

Material examined $(4 \stackrel{+}{+}, 7$ すへ)

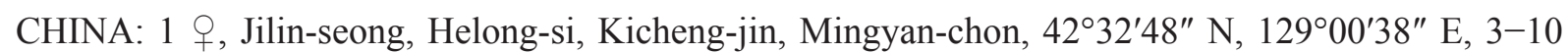
Jul. 2009, MT, J.W. Lee leg. (YNU); 1 స̂, Jilin-seong, Helong-si, Xicheong-jin, Mingyan-chon, $42^{\circ} 32^{\prime} 48^{\prime \prime} \mathrm{N}, 129^{\circ} 00^{\prime} 38^{\prime \prime}$ E, 15-22 Jun. 2009, MT, J.W. Lee leg. (YNU).

FRANCE: 1 \&, Lot, Cahors, 1-13 Jul. 1990, YPT, H.Tussac leg. (CNCI).

JAPAN: 2 q $q$, Hokkaido, Lake Utonai, alt. 10 m, 6 Jul. 1989, M.J. Sharkey leg., sweep (CNCI); 2 §ิ ô, Hokkaido, Sapporo, 25 Jun.-2 Jul. 1987, MT, K. Maeto leg. (CNCI); 1 ð’, same collecting data, 18-21 ? 1987, MT, K. Maeto leg. (CNCI); 1 ô, same collecting data, 24-29 Jul. 1988, MT, K. Maeto leg. (CNCI); 1 §ै, same collecting data, 18-24 Jun. 1989, MT, K. Maeto leg. (CNCI).

SOUTH KOREA: 1 ठ, National DMZ Native Botanic Garden, Yanggu-gun, Gangwon Province, 30 May 2015, I.K. Kim leg. (KNA).

\section{Variation}

Body length $2.00-3.17 \mathrm{~mm}$ in both sexes; male antenna uniformly bright yellow or yellow with apical segments one to four darkened; male hind tibia brown to dark brown.

\section{Distribution}

China (Jilin) (new record), France (new record), Japan (Hokkaido) (new record), South Korea (new record), Russia (Far East) (Kolyada \& Chemyreva 2016).

Ismarus brevis Kim \& Lee sp. nov. urn:1sid:zoobank.org:act:C5CF9B40-BF24-4EB6-9CEA-9AAF4B22BA88

Fig. 3

\section{Diagnosis}

Ismarus brevis sp. nov. is quite distinct from other described Palaearctic species in antenna length and antennal segment ratios. The very short antenna and quadrate A5-A13 are distinct characters among Palaearctic Ismarus.

\section{Etymology}

The specific epithet brevis is derived from the Latin adjective which means short.

Type material $(5$ ふぶ)

\section{Holotype}

SOUTH KOREA: $\widehat{\jmath}$, Gyeongsangbuk Province, Mungyeong-si, Gaeun-eup, Wonjang-ri, Mt. Songnisan, Beorimgijae, 3640'59" N, 12757'07" E, 21 May-15 Jun. 2013, J.K. Choi leg. (YNU). 

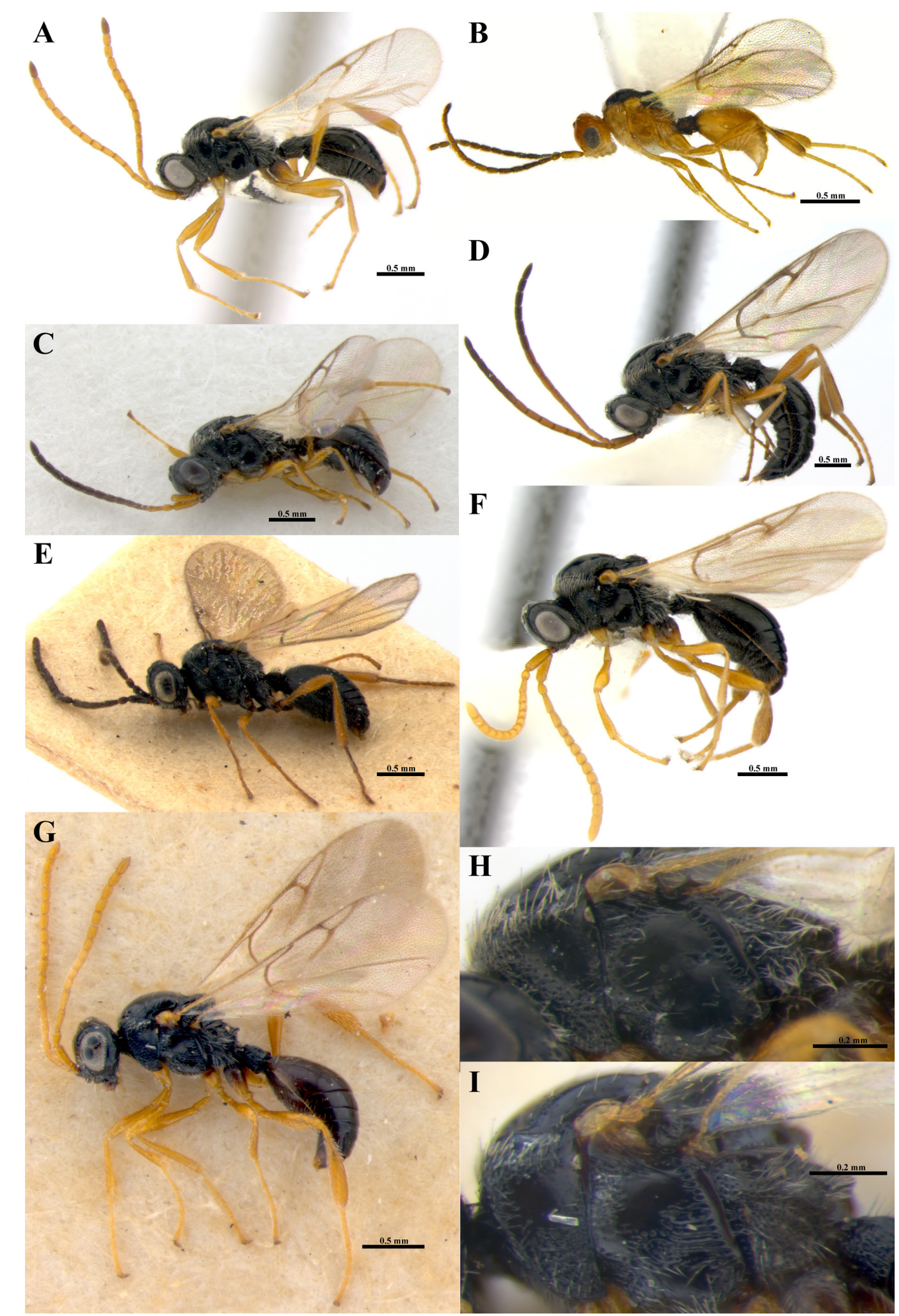

Fig. 1. Ismarus spp., 우 (A-G. Habitus; H-I. Mesosoma in lateral view). A. I. apicalis Kolyada \& Chemyreva, 2016. B. I. dorsiger (Haliday, 1831). C, H. I. halidayi Förster, 1850. D. I. grandis Alekseev, 1978. E. I. rugulosus Förster, 1850. F. I. spinalis Kolyada \& Chemyreva, 2016. G, I. I. flavicornis (Thomson, 1858). 

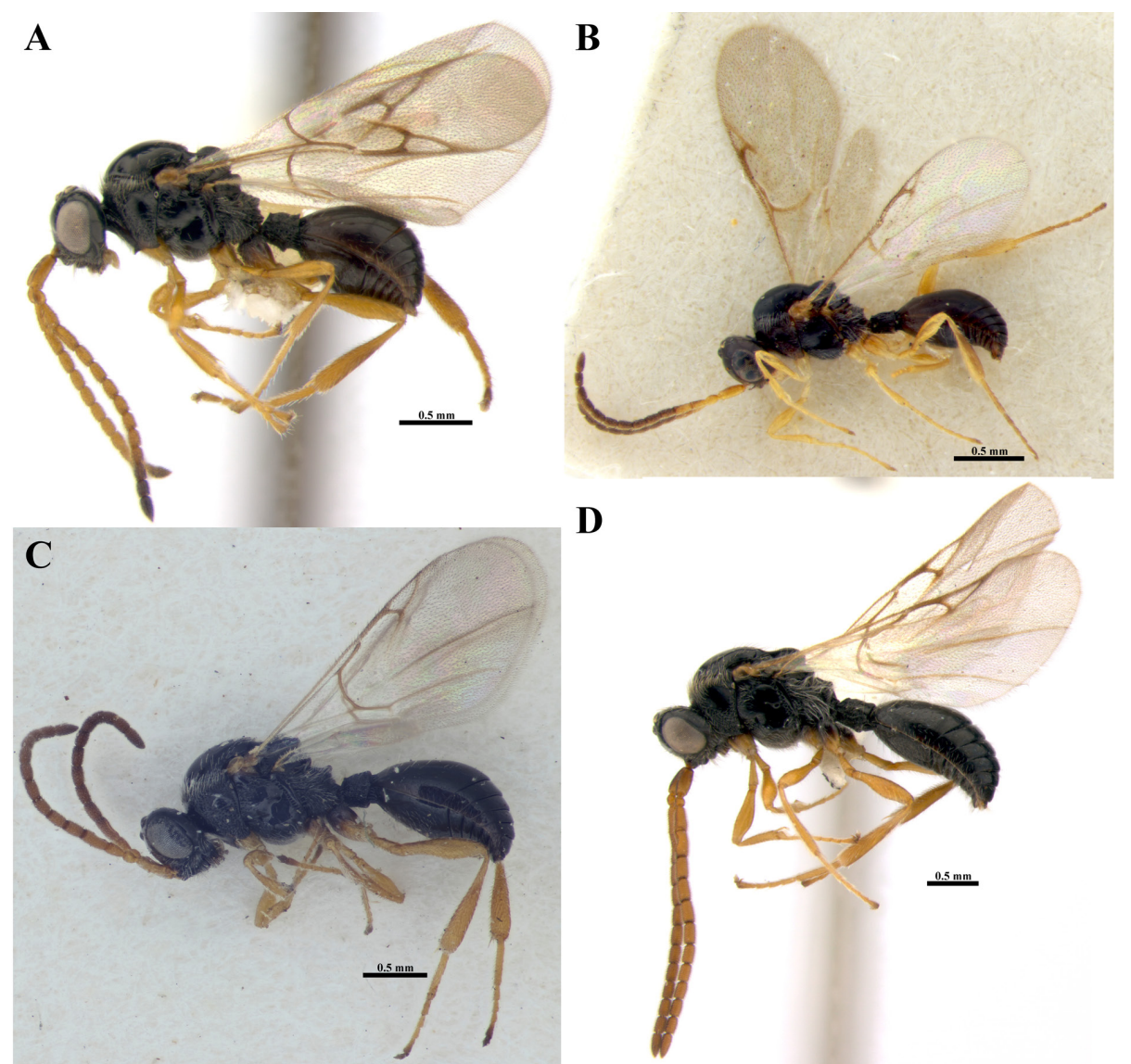

D
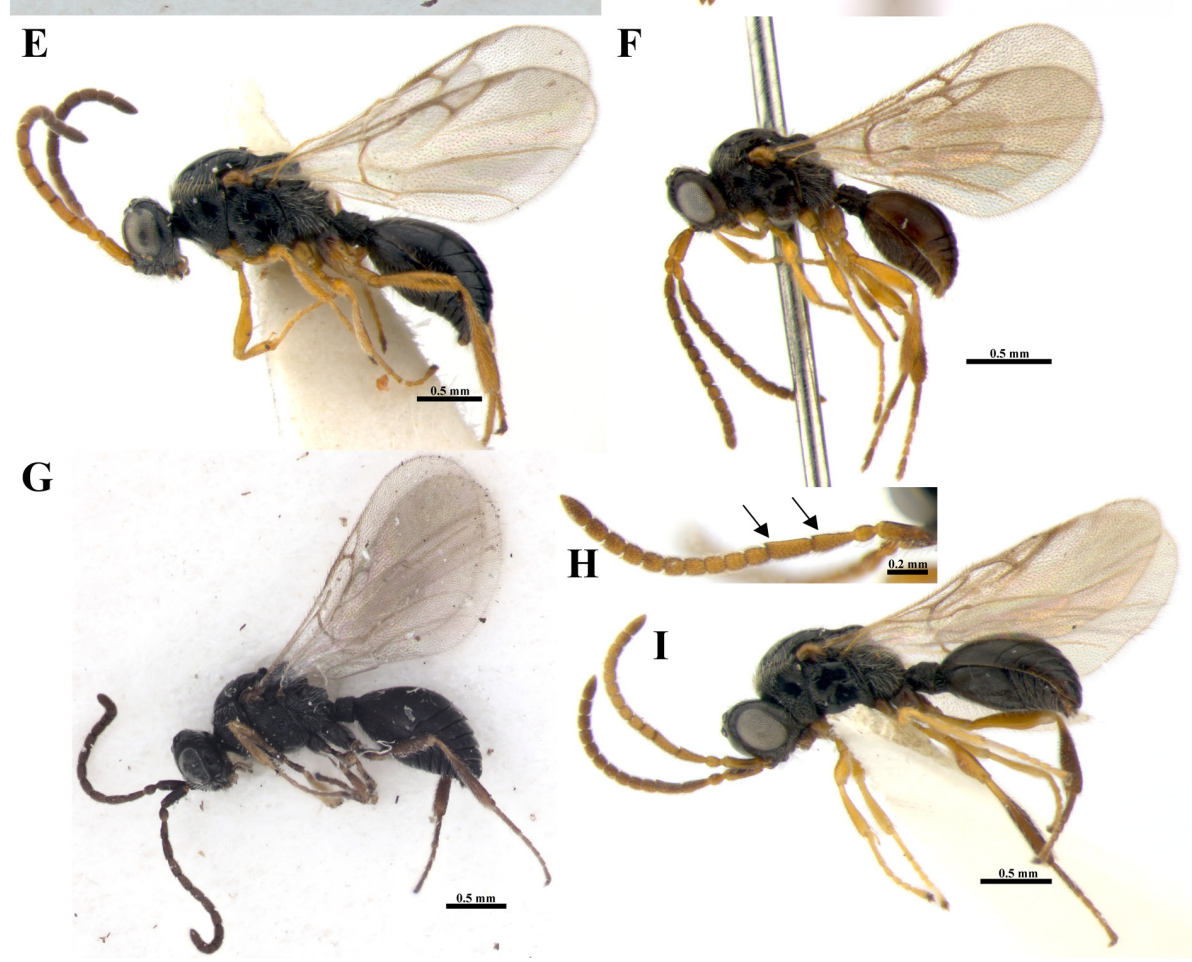

Fig. 2. Ismarus spp., $\widehat{\jmath}$. (A-G, I. Habitus; H. Antennae). A. I. apicalis Kolyada \& Chemyreva, 2016. B. I. dorsiger (Haliday, 1831). C. I. flavicornis (Thomson, 1858). D. I. grandis Alekseev, 1978. E. I. halidayi Förster, 1850. F. I. multiporus Kolyada \& Chemyreva. G. I. rugulosus Förster, 1850. H-I. I. spinalis Kolyada \& Chemyreva, 2016. 
Paratypes

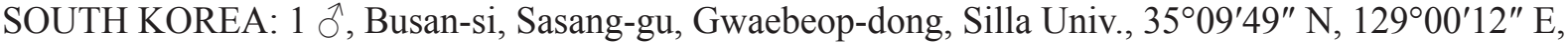
7-22 May 2008, MT, J.W. Lee leg. (YNU); 1 §, Gyeongsangbuk Province, Cheongdo-gun, Unmun-

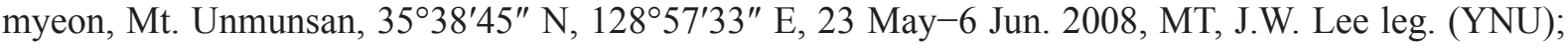

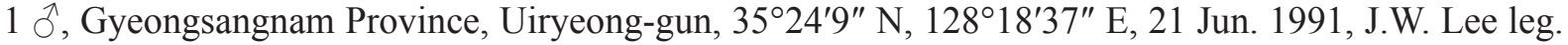
(YNU).

RUSSIA: 1 đ̊, Far East, env. Vladivostok, Jul. 1992, A.Okulov leg., swept (CNCI).

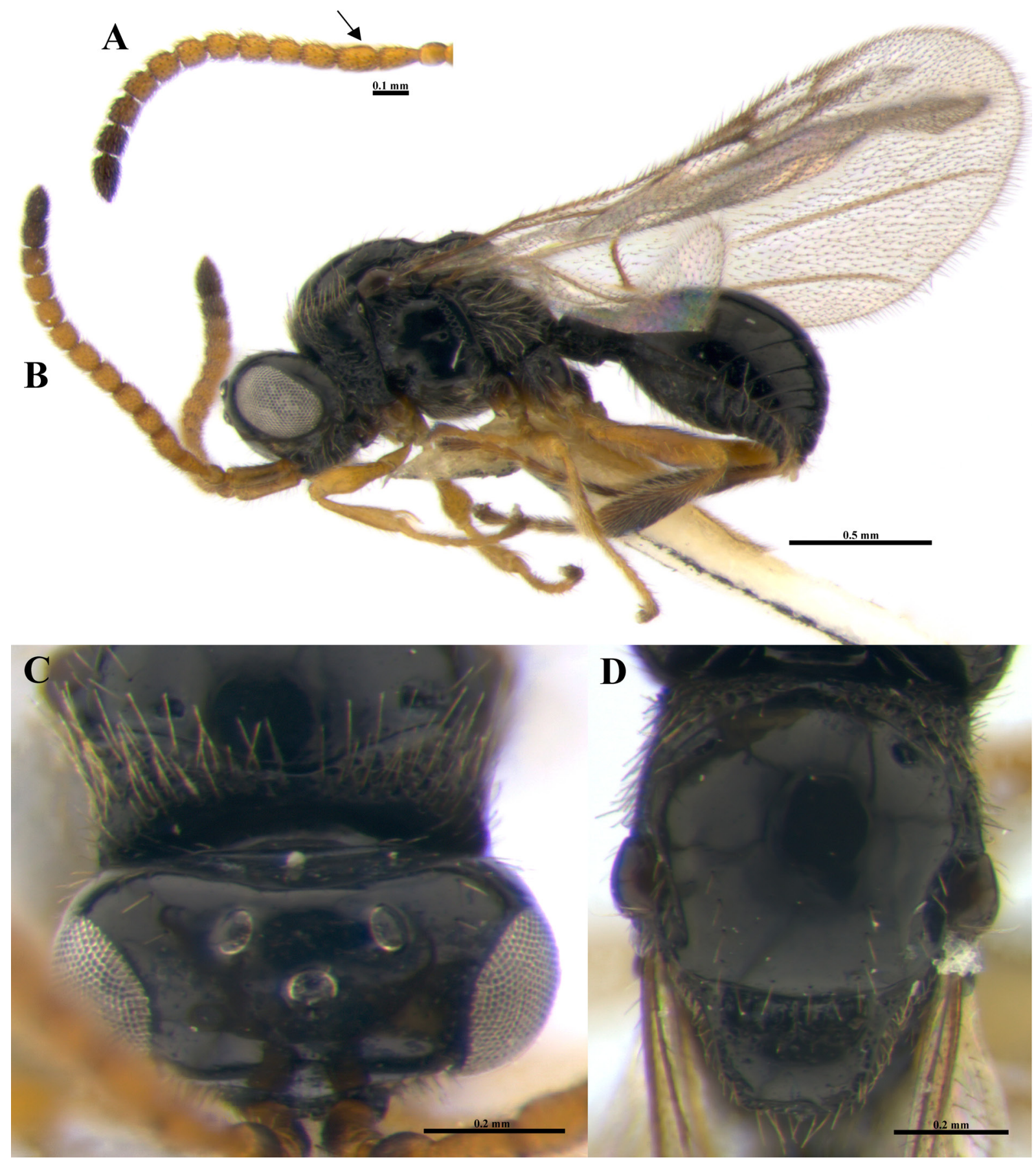

Fig. 3. Ismarus brevis Kim \& Lee sp. nov., holotype, ô. A. Antenna. B. Habitus in lateral view. C. Head in dorsal view. D. Mesosoma in dorsal view. 


\section{Description}

Male (holotype)

HEAD. Head in dorsal view much wider than long $(58: 32)$, slightly wider than width of mesosoma (58:48); POL: 13; LOL: 6; OOL: 10 (Fig. 3C); ocelli large, LOL slightly longer than diameter of lateral ocellus $(6: 5)$; vertex behind ocelli nearly flat in lateral view; eye large and without setae; inner orbits, frons and temple with few sparse setae; above antennal sockets, face and cheek with numerous long setae; antenna much shorter than body length $(2: 3)$; scape and pedicel with scattered setae, A3-A15 with dense and short setae; blade-like carina on A4, basal $0.7 \times$ length of segment (Fig. 3A); antennal segments in following proportions (length:width): $18: 6 ; 7: 8 ; 11: 5 ; 10: 6 ; 8: 7 ; 8: 7 ; 7: 7 ; 7: 7 ; 7: 7$; $7: 7 ; 7: 7 ; 7: 7 ; 7: 7 ; 11: 7$ (Fig. 3A).

Mesosoma. Pronotum in dorsal view coarsely punctate and whitish long setae along the posterior margin; pronotal shoulders angled; upper part of lateral pronotum predominantly smooth and concave in the middle except upper margin coarsely punctate, lower part of lateral pronotum with irregular transverse striae; mesoscutum smooth and convex; notauli present anteriorly as large pits (Fig. 3D); humeral sulcus deep, longer than length of tegula $(5: 4)$; scutellum smooth and slightly convex, posterior rim rounded (Fig. 3D); anterior scutellar pit large and deep, much shorter than remaining scutellar disc, nearly smooth at bottom, median keel weak (Fig. 3D); mesopleuron predominantly smooth with deep crenulate line along posterior margin (Fig. 3B); metapleuron rugose and covered with dense long setae.

WINGS. Fore wing with costal, subcostal, basal, marginal, postmarginal, radial and stigma veins tubular; medial vein pigmented; radial cell closed, as long as marginal vein and $3.1 \times$ its height.

LEGS. Fore and mid legs slender; hind tibiae gradually swollen.

Metasoma. Petiole short and expanded (2:3), irregular longitudinal carinae dorsally; tergites completely smooth, with scattered setigerous punctures; base of second tergite with several short costae basally and long and deep median furrow, extending $0.60 \times$ length of second tergite; sutures between tergites complete and deeply impressed.

CoLour. Body black; antennae yellowish brown except apical segment brown; legs and tegulae yellowish brown to brown; wings hyaline, covered with brown setae.

Measurements. Head length $0.43 \mathrm{~mm}$, width $0.73 \mathrm{~mm}$; mesosoma length $0.90 \mathrm{~mm}$, width $0.62 \mathrm{~mm}$; metasoma length $1.02 \mathrm{~mm}$; fore wing length $2.41 \mathrm{~mm}$; total body length $2.35 \mathrm{~mm}$.

\section{Female}

Unknown.

\section{Variation}

Body length $2.00-2.61 \mathrm{~mm}$; body colour dark brown to black, antenna yellowish brown with A15 or A14-A15 or A12-A15 dark brown; median furrow extending $0.60-0.75 \times$ length of second tergite.

\section{Host}

Unknown.

\section{Distribution}

Russia (Primorsky Krai), South Korea. 
Ismarus distinctus Kim, Notton \& Ødegaard sp. nov. urn:1sid:zoobank.org:act:9654D5C2-C637-4102-93B8-EABD2FD6C45A

Fig. 4

\section{Diagnosis}

The punctured scutellum and weakly rugulose tergites are distinct characters among Palaearctic Ismarus.

\section{Etymology}

The specific name distinctus is derived from the Latin adjective, meaning distinct.

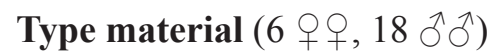

\section{Holotype}

NORWAY: +, EIS 37, AK (Akershus), Skedsmo, Asakmoen, 59.98538 ${ }^{\circ} \mathrm{N}, 11.11037^{\circ} \mathrm{E}$, 27 Jul.-21 Aug. 2010, MT, F. Ødegaard leg. (NINA).

\footnotetext{
Allotype

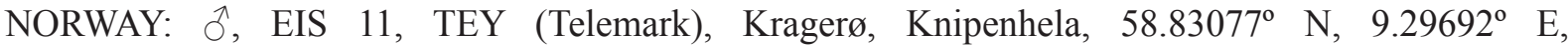
16 Jun.-14 Jul. 2015, MT, F. Ødegaard leg. (YNU).

\section{Paratypes}

NORWAY: 1 +, EIS 37, AK (Akershus), Skedsmo, Asakmoen, 59.98538 N, $11.11037^{\circ} \mathrm{E}$, 27 Jul.-21 Aug. 2010, MT, F. Ødegaard leg. (NINA); 1 §ิ, EIS 28, AK (Akershus), Oslo, Bleikøya, $59.88968^{\circ} \mathrm{N}, 10.74285^{\circ}$ E, 26 Jun.-28 Jul. 2009, MT, A. Endrestøl leg. (NINA); 1 ○, EIS 19, VE (Vestfold), Larvik, Stavern, Agnes, $59.01560^{\circ} \mathrm{N}, 10.02295^{\circ}$ E, 12 Jul.-14 Aug. 2012, MT, F. Ødegaard leg. (NINA); $1 \delta^{\lambda}$, EIS 19, VE, Horten, Borrevann, Horten natursenter, $59.41715^{\circ} \mathrm{N}, 10.43856^{\circ} \mathrm{E}$,

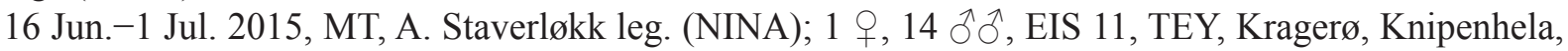

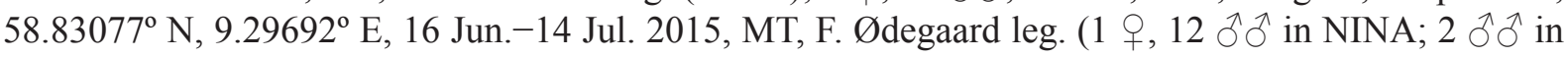

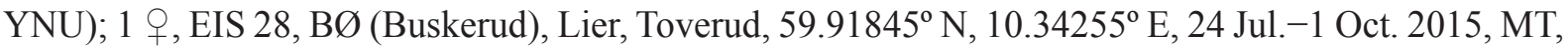
F.Ødegaard leg. (NINA).
}

UNITED KINGDOM: 1 , , England, Norfolk, Santon Downham, TL818883, MT, heath with Betula \& Pinus, 20-30 Jul. 1985, J. Field leg. (DNPC); 1 \&, England, Surrey, Kew, Populus italica, 22 Jul. 1979, V.F. Eastop leg. (DNPC).

\section{Description}

Female (holotype)

HEAD. Head in dorsal view much wider than long (16:9), slightly wider than width of mesosoma $(8: 7)$; POL: 5; LOL: 4; OOL: 3 (Fig. 4E); ocelli large, LOL slightly longer than diameter of lateral ocellus (9:7); vertex behind ocelli nearly flat in lateral view; eye large and without setae; inner orbits, frons and temple with few sparse setae; above antennal sockets, face and cheek with numerous long setae; antenna much shorter than body length (11:14); scape and pedicel with scattered setae; A3-A15 with dense and short setae; antennal segments in following proportions (length:width): 38:13; 20:10;22:9; 26:9; $22: 9 ; 22: 9 ; 20: 10 ; 20: 10 ; 18: 11 ; 18: 11 ; 18: 11 ; 18: 11 ; 18: 11 ; 18: 11 ; 28: 11$ (Fig. 4D).

Mesosoma. Pronotum in dorsal view coarsely punctate with whitish long setae; pronotal shoulders angled; upper part of lateral pronotum predominantly smooth and concave in the middle except anterior and upper margin coarsely punctate, lower part of lateral pronotum punctate-rugose; mesoscutum smooth and convex; notauli present anteriorly as large pits; humeral sulcus deep and long, longer than length of tegula (19:13); scutellum punctate to rugose and slightly convex, posterior rim rounded (Fig. 4D-E); anterior scutellar pit large and deep, shorter than rest of scutellar disc, strongly crenulate at bottom, 
median keel indistinct; mesopleuron predominantly smooth with deep crenulate line along posterior margin; metapleuron rugose and covered with dense long setae.

WINGS. Fore wing with costal, subcostal, basal, marginal, postmarginal, radial and stigmal veins tubular; medial vein pigmented; radial cell closed, $0.70 \times$ as long as marginal vein and $3.0 \times$ its height (Fig. 4D).

LEGS. Fore and mid legs slender; hind tibiae gradually swollen.

Metasoma. Petiole short and expanded (2:3), with strong costae dorsally; tergites weakly rugulose, with scattered setigerous punctures; base of second tergite with several short costae basally and median furrow deep basally to shallow apically, extending over half of second tergite (Fig. 4D); sutures between tergites complete and deeply impressed.
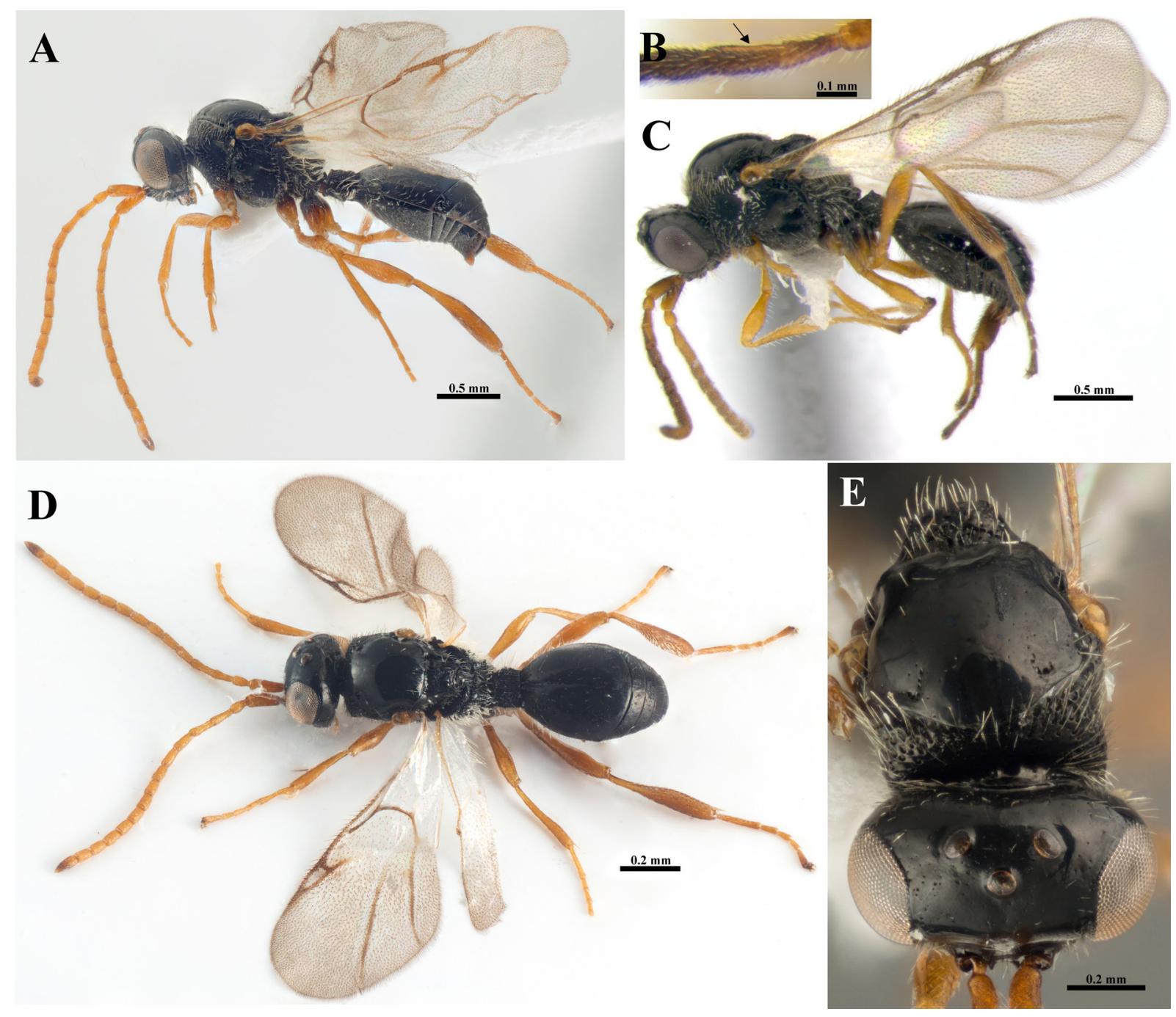

Fig. 4. Ismarus distinctus Kim, Notton \& Ødegaard sp. nov. (A, D-E. Holotype, ค; B-C. Allotype, Љ゙). A. Habitus in lateral view. B. Antenna (A3-A5). C. Habitus in lateral view. D. Habitus in dorsal view. E. Head, Mesosoma in dorsal view. 
CoLour. Body black; antennae uniformly bright yellow except for apical segment brownish; legs and tegulae yellow, except basal half of hind coxae black to dark-brown, hind tibiae yellowish brown; wings hyaline, covered with brown setae.

Measurements. Head length $0.57 \mathrm{~mm}$, width $0.78 \mathrm{~mm}$; mesosoma length $1.13 \mathrm{~mm}$, width $0.70 \mathrm{~mm}$; metasoma length $1.34 \mathrm{~mm}$; fore wing length $2.65 \mathrm{~mm}$; total body length $3.04 \mathrm{~mm}$.

Male (allotype)

Body length $2.78 \mathrm{~mm}$. Similar to female, but scape and pedicel yellowish brown except dorsal part of scape dark brown, antennomeres brown, legs yellowish brown except hind tibiae and tarsus brown (Fig. 4C); base of second tergite with several short costae basally and median furrow deep basally to shallow apically, extending $0.65 \times$ length of second tergite; blade-like carina on A4 percurrent (Fig. 4B); antennal segments in following proportions: $18: 6 ; 10: 6 ; 12: 5 ; 15: 6 ; 10: 6 ; 10: 6 ; 10: 6 ; 10: 6 ; 10: 6$; $9: 6 ; 9: 6 ; 9: 6 ; 9: 6 ; 16: 6$; hind tibia slender.

\section{Variation}

Body length $2.34-3.04 \mathrm{~mm}$ in both sexes; median furrow extending $0.6-0.7 \times$ length of second tergite in both sexes; the strength of the rugosity of the tergites varies from weak to very weak, but it is always visible.

\section{Host}

Unknown.

\section{Distribution}

Norway, United Kingdom.

Ismarus dorsiger (Haliday, 1831)

Figs 1B, 2B

Cinetus dorsiger Haliday in Curtis, 1831: 380.

Belyta anomala Nees von Esenbeck, 1834: 345. Synonymized by Förster (1856).

Ismarus neesii Förster, 1850: 286. Synonymized by Haliday (1857).

Ismarus moravicus Ogloblin, 1925: 50. Synonymized by Kolyada \& Chemyreva (2016).

Ismarus dorsiger - Haliday 1835: 467. Generic transfer.

\section{Diagnosis}

Female body white to yellow except mesoscutum, scutellum and occasionally petiole black, male body black with yellow legs; mandibles whitish to yellow in both sexes; mesoscutum without notauli in both sexes; radial cell much shorter than length of marginal vein in both sexes.

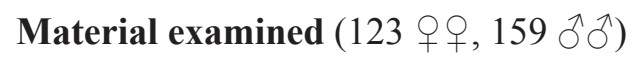

FRANCE: 1 q, Finistère, Morlaix, 11 Jun. 1954, J.F. Perkins leg. (NHMUK010264876). GERMANY: 1 ㅇ, Baden-Württemberg, nr Lautenbach, River Rench, 30 Mar. 1992, M. Boness leg. (DNPC); 1 ㅇ, North Rhine-Westphalia, Leverkusen, Bergisch-Neukirchen, River Wupper, reared from flood debris, 19 Jan. 1984, M. Boness leg. (DNPC); 1 q, same collecting data, 9 Mar. 1999 (DNPC); 1 + , same collecting data, 20 Feb. 1999 (DNPC); 1 +, same collecting data, 24 Mar. 2000 (DNPC); 1 , same collecting data, 15 Feb. 2001 (DNPC); 1 , , same collecting data, 14 Mar. 2004 (NHMUK010264879); 1 q, North Rhine-Westphalia, Leverkusen, Hitdorf, River Rhine, 22 Feb. 1995, M. Boness leg. 
(DNPC); 1 , , North Rhine-Westphalia, Leverkusen, Wiesdorf, River Dhunn, reared from flood debris, 8 Apr. 1970, M. Boness leg. (DNPC); 1 o, Rhineland-Palatinate, Ahrweiler district, Bad Breisig, at window, 3 Jan. 2006, M. Boness leg. (NHMUK010264880).

MONTENEGRO: 1 q, Durmitor Mountains, Žabljak Municipality, Dobrilovina, 12 Sep. 1984, Z. Bouček leg. (NHMUK010264878).

NORWAY: 1 , Akershus, Oslo, Bleikøya, 26 Jun.-28 Jul. 2009, MT, A. Endrestøl leg. (NINA); 1 , Akershus, Aurskog-Høland, Bråten, 10 Aug.-14 Sep. 2015, MT, A. Staverløkk leg. (NINA); 6 우, Vestfold, Horten, Østøya, 1 Jul.-12 Aug. 2015, MT, A. Staverløkk leg. (NINA); 3 qo+, Telemark, Siljan, Brenndalskarven, 8 Aug.-1 Oct. 2015, MT, F. Ødegaard leg. (NINA); 1 q, Telemark, Kragerø, Grønåsliane, 14 Jul.-18 Aug. 2015, MT, F. Ødegaard leg. (NINA); 1 , Vest-Agder, Nedre Timenes, 1 Aug. 2015, MT, A. Staverløkk leg. (NINA); 1 ㅇ, Vest-Agder, Birkenes, Mollestad, 4-24 Jul. 2016, MT, A. Staverløkk leg. (NINA); 1 +, Hordaland, Masfjorden, Stormyra, 25 Aug.-23 Sep. 2016, MT, A. Staverløkk leg. (NINA); 1 q, Møre og Romsdal, Norddal, Løberget, 24 Aug.-30 Sep. 2015, MT, O. Hanssen leg. (NINA).

REPUBLIC OF IRELAND: 1, sex unknown, Co. Sligo, Trawalua, 24-29 Jul. 1933, G.E.J. Nixon leg. (NHMUK010264865).

SOUTH KOREA: 1 क , National DMZ Native Botanic Garden, Yanggu-gun, Gangwon Province, 30 Jun. 2015, I.K.Kim (KNA); 2 +, , same collecting data, 15 Sep. 2015, I.K. Kim leg. (KNA).

SWEDEN: 1 †, Skåne, Åhus, 14 Jul. 1974, K.-J. Hedqvist leg. (NHMUK010264870); 3 qo , Skåne, Haväng, 28 Jul. 1973, K.-J. Hedqvist leg. (NHMUK010264868, NHMUK010264871, NHMUK010264874); 2 우, Skåne, Knäbäck, 15 Jul. 1973, K.-J. Hedqvist leg. (NHMUK010264869, NHMUK010264872); 2 우, Skåne, Skepparpsgarden, 17 Jul. 1971, K.-J. Hedqvist leg. (NHMUK010264867, NHMUK010264875); 1 †, Skåne, Södra Sandby, Måryd, Aug. 1976, T. Huddleston and J. Quinlan leg. (NHMUK010264866); 1 ㅇ, Uppland, Vallentuna, 17 Aug. 1974, K.-J. Hedqvist leg. (NHMUK010264873).

SWITZERLAND: 1 q, Zürich, Dielsdorf, old coniferous forest, 3 Aug. 1984, L. Masner leg. (NHMUK010264877).

UNITED KINGDOM: 1 , England, Beds, Aspley Heath, SP927348, 14 Aug. 1960, (NHMUK 010264822); 1 Ô,, England, Beds, Barton Hills, TL089297, 25 Jul. 1970, V.H. Chambers leg. (NHMUK010264835); 1 ð̊, same collecting data, 27 Jul. 1973 (NHMUK010264847); 1 , , England, Beds, Chicksands, TL125389, 15 Aug. 1974, V.H. Chambers leg. (NHMUK010264815); 1 , , same collecting data, 20 Jul. 1976 (NHMUK010264816); 1 9, England, Beds, Clophill, TL081377, 25 Aug. 1956, V.H. Chambers leg. (NHMUK010264819); 1 ô, England, Beds, Dunton, TL237442, 15 Jul. 1973, V.H. Chambers leg. (NHMUK010264838); 1 ô, same collecting data, 22 Jul. 1973 (NHMUK010264844); 1 ô, same collecting data, 22 Jul. 1974 (NHMUK010264842); 1 ô, same collecting data, 28 Jul. 1974 (NHMUK010264843); 1 ô, same collecting data, 5 Aug. 1974 (NHMUK010264845); 1 \&, England, Beds, Edworth, TL226410, 15 Aug. 1971, V.H. Chambers leg. (NHMUK010264814); 1 ô, same collecting data, 26 Jul. 1971 (NHMUK010264849); 1 क, England, Beds, Eversholt, SP996330, 10 Sep. 1960, V.H. Chambers leg. (NHMUK010264821); 1 त̃, England, Beds, Flitwick Moor,

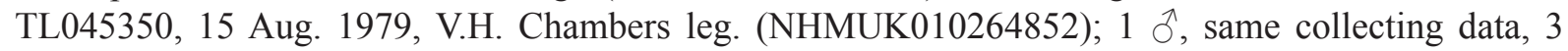

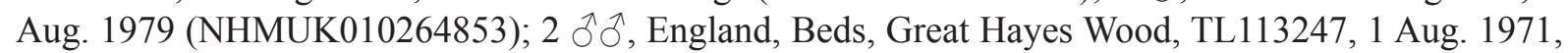
V.H. Chambers leg. (NHMUK010264837, NHMUK010264850); 1 స, England, Beds, Long Lane, Toddington, TL014300, Acer pseudoplatanus, 3 Aug. 1957, V.H. Chambers leg. (NHMUK010264839); 1 q, England, Beds, Maulden, TL050380, 28 Aug. 1974, V.H. Chambers leg. (NHMUK010264818); 
1 ô, same collecting data, 11 Aug. 1972 (NHMUK010264846); 1 ㅇ, England, Beds, Steppingly, TL010353, 26 Sep. 1971, V.H. Chambers leg. (NHMUK010264817); 1 त, same collecting data, 15

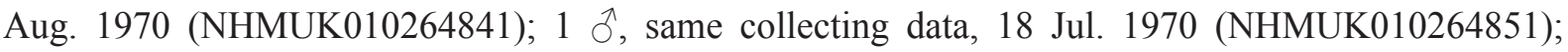
2 ठ̊̊, England, Beds, Sutton Fen, TL202475, 18 Jul. 1978, V.H. Chambers leg. (NHMUK010264854, NHMUK010264855); 1 ð, same collecting data, 21 Jul. 1980 (NHMUK010264836); 1 ð, same collecting data, 27 Jul. 1978 (NHMUK010264840); 1 +, same collecting data, 27 Jul. 1978 (NHMUK010264820); 1 ô, England, Beds, 8 Aug. 1972, V.H. Chambers leg. (NHMUK010264848); 1 ô, England, Berks, Thatcham Moor, 27 Jul. 1975, J.S.Noyes (NHMUK010264856); 1 ภ, 2 웅, England, Bucks, Burnham Beeches, SU98, fogging, Fagus sylvatica, 1990, H. Read leg. (DNPC); 1 ô, England, Bucks, Burnham Beeches, beating and sweeping, 24 Jul. 1975 (NHMUK010264834); 1 ô, England, Cambs, Cambridge, Jun. 1984, P.F. Yeo leg; (NHMUK010264833); 2 우, England, Cambs, Chippenham Fen, TL650693, carr/ reedbed, 22 Aug. -5 Sep. 1985, MT, Field leg. (DNPC); 1 §, same collecting data, 26 Jul.-10 Aug. 1983 (DNPC); 1 ठૈ, same collecting data, 29 Jun.-9 Jul. 1984 (DNPC); 1 ภ, same collecting data, 1 Aug. 1984 (DNPC); 1 +, England, Cambs, Duxford, 25 Jul.-1 Aug. 1979, R.S. George leg. (NHMUK010264823); 1 ô, England, Ches, Abbotts Moss, SJ596680, stream, 10 Jul. 1990, swept, D.G.

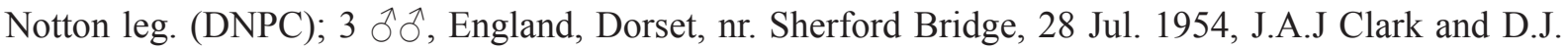
Clark leg. (NHMUK010264829, NHMUK010264830, NHMUK010264831); 3 우, England, Hants, New Forest, Round Hill, 10 Aug. 1975, Z. Bouček leg. (NHMUK010264811, NHMUK010264812, NHMUK010264813); 1 \&, England, Hants, Whitley Wood, SU2905, litter, 15-16 Jul. 2002, P. Eggleton et al. leg. (NHMUK010264827); 1 q, same collecting data, 19-20 Sep. 2002 (NHMUK010264826); 1 đ, England, Kent, Murston, TQ928648, 16 Sep. 1983, L. Clemons leg. (NHMUK010264861); 1 \%, England, Lincs, Tetford Hill, 17 Jul. 1951, M.W.R. de V. Graham leg. (NHMUK010264859); 1 ㅇ, England, Middx, Southgate, 13 Aug. 1964, M.W.R. de V. Graham leg. (NHMUK010264825); 1 ô, same collecting data, 18 Jul. 1968 (NHMUK010264860); 1 ô, England, Norfolk, East Wretham Nature Reserve, TL98, MT, 14 Jul. 1974, L. Rogers, M.G. Fitton and M.C. Day leg. (NHMUK010264832);

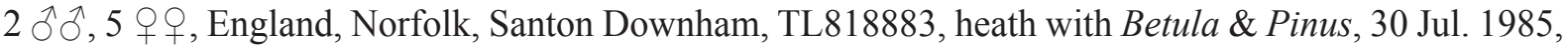

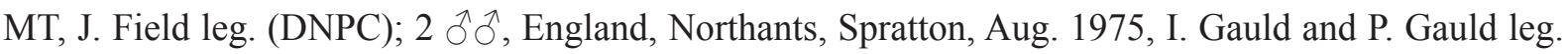
(NHMUK010264857, NHMUK010264858); 1 ㅇ, England, Oxon, near Cothill, Acer pseudoplatanus, 11 Sep. 1989, J.W. Ismay leg. (DNPC); 1 ô, same collecting data, 13 Aug. 1989 (DNPC); 1 ô, England, Oxon, Shiplake, by River Thames, SU770783, 6 Jul. 1990, D.G. Notton leg. (DNPC); 1 , England, Oxon, Wytham, 29 Aug. 1962, M.W.R. de V. Graham leg. (NHMUK010264828); 1 q, England, Somerset, Bicknoller, ST103383260898, date unknown, A.G. Smith and P. Hill-Cottingham

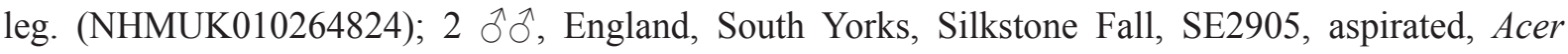
pseudoplatanus, 21 Aug. 1991, D.G. Notton leg. (DNPC); 1 đ̃, England, Surrey, Barnes Common,

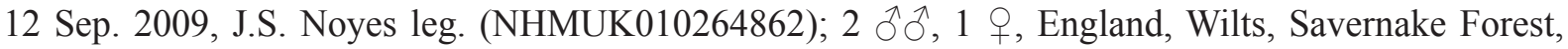

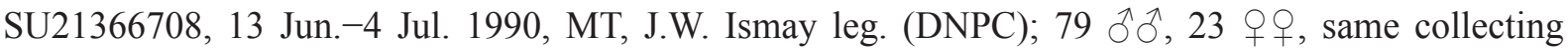

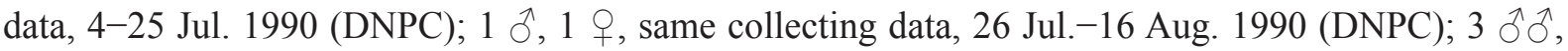
England, Wilts, Savernake Forest, SU22906558, 13 Jun.-4 Jul. 1990, MT, J.W. Ismay leg. (DNPC);

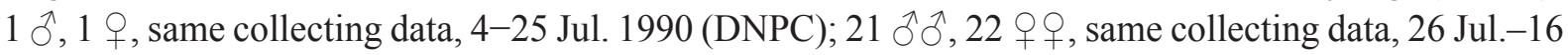
Aug. 1990 (DNPC); 2 우, same collecting data, 15 Aug. -5 Sep. 1990 (DNPC); 1 §, same collecting data, 26 Sep. -17 Oct. 1990 (DNPC); 1 ô, Scotland, Highland, Beinn Eighe, NG96, Aug. 1988, I. MacGowan

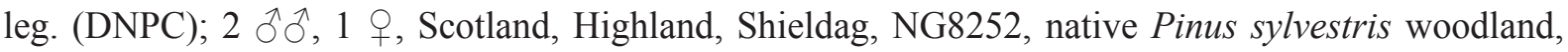
Aug. 1991, I. MacGowan leg. (DNPC); 1 đ̂, 1 ㅇ, Scotland, Moray, Culbin Forest, NH9458, Aug. 1992, I. MacGowan leg. (DNPC); 1 §̃, Scotland, Perth and Kinross, Ballinluig, 20 Jul. 1977, J.S. Noyes, L. Rogers and T. Huddleston leg. (NHMUK010264864); 1 $\curvearrowright$, Wales, Rhondda Cynon Taf, Pontypridd, 25 May 1975, J.S. Noyes leg. (NHMUK010264863); 1 ㅇ, Wales, Wrexham, Trevor, 15 Sep. 1977, Z. Bouček leg. (NHMUK010209576). 


\title{
Host
}

Hyperparasitoid of Aphelopus serratus Richards, 1939 (Dryinidae) in Italy (Olmi 2000). In addition, we have excluded the reference of Waloff \& Jervis (1987). Because they were not sure about the identity of Aphelopus melaleucus (Dalman, 1818), their identification was "probable", as written by Jervis (1979) (Olmi, pers. comm.).

\section{Distribution}

France (new record), Montenegro (new record), Norway (new record); South Korea (new record), Switzerland (new record), Andorra (Ventura et al. 1997), Bulgaria (Petrov 1990), China (Yunnan) (Liu et al. 2011), Czech Republic (Ogloblin 1925), Denmark (Johnson 2016), Finland (Hellén 1964), Germany (Hellén 1964), Italy (Bin et al. 1995), the Netherlands (Peeters 2015), Republic of Ireland (Nixon 1957; Stelfox 1966; O'Connor et al. 2004); Russia (European) (Kolyada \& Chemyreva 2016), Spain (Martínez de Murguía 1998), Sweden (Thomson 1858; Hellén 1964), United Kingdom (Nixon 1957; Notton 1996; O’Connor et al. 2004).

This species, widely distributed in Europe to Oriental China (Yunnan), is now recorded for the first time from the Eastern Palaearctic (South Korea)

\section{Variation}

All European specimens have a yellow petiole, but Chinese and South Korean specimens have a black petiole.

\author{
Ismarus excavatus Kim \& Lee sp. nov. \\ urn:1sid:zoobank.org:act:1BFEA38D-668E-43F6-9344-31427751E70A
}

Fig. 5

\section{Diagnosis}

Ismarus excavatus sp. nov. is quite distinct from other described Palaearctic species in the male sex segment; A4 is distinctly excavate, and curved.

\section{Etymology}

This species is named excavatus in recognition of the strongly excavated male A4.

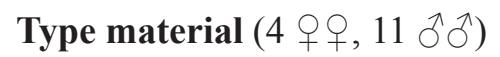

\section{Holotype}

JAPAN: + , Aomori, Tsuta, Onsen area, alt. 500 m, 40¹0' N, 14057’ E, 22 Aug. 1996, L. Masner leg. (CNCI).

\footnotetext{
Allotype

JAPAN: $\widehat{\partial}$, same collecting data as for holotype (CNCI).

\section{Paratypes}

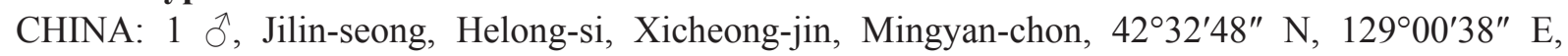
8-15 Jun. 2009, MT, J.W. Lee leg. (YNU); 1 đ̃, same collecting data, 15-22 Jun. 2009, MT, J.W. Lee leg. (YNU); $5 \hat{\partial} \hat{\partial}$, same collecting data, 22-29 Jun. 2009, MT, J.W. Lee leg. (YNU); $1 \hat{\partial}$, same collecting data, 29 Jun.-6 Jul. 2009, MT, J.W. Lee leg. (YNU).
}

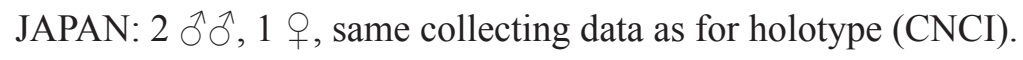


SOUTH KOREA: 1 q, Chungcheongbuk Province, Boeun-gun, Songnisan-myeon, Sanae-ri 209,

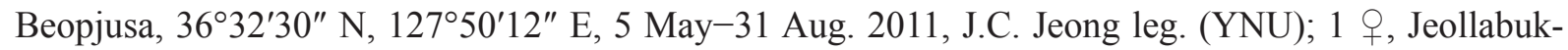

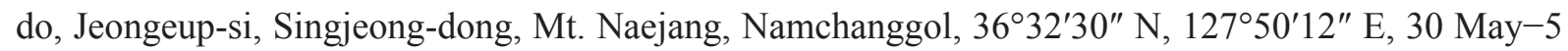
Aug. 2011, NT, J.W. Lee leg. (YNU).

\section{Description}

Female (holotype)

HeAD. Head in dorsal view much wider than long $(13: 7)$, slightly wider than width of mesosoma $(13: 11)$; POL: 14; LOL: 7; OOL: 10 (Fig. 5C); ocelli large, LOL slightly longer than diameter of lateral ocellus (7:6); vertex behind ocelli nearly flat in lateral view; eye large and without setae; inner orbits, frons and temple with few sparse setae; above antennal sockets, face and cheek with numerous long setae; antenna shorter than body length (3:4); scape and pedicel with scattered setae; A3-A15 with dense and short setae; antennal segments in following proportions (length: width): $21: 6 ; 10: 5 ; 12: 4 ; 13: 5 ; 10: 5 ; 10: 6$; $10: 7 ; 10: 7 ; 10: 7 ; 10: 7 ; 9: 7 ; 9: 7 ; 8: 7 ; 8: 7 ; 15: 7$ (Fig. 5A).

Mesosoma. Pronotum in dorsal view punctate-rugose with whitish long setae along the posterior margin; pronotal shoulders angled; lateral pronotum predominantly punctate to punctate-rugose except smooth and concave in the middle; mesoscutum smooth and convex; notauli present anteriorly as large pits (Fig. 5D); humeral sulcus fine, longer than tegula (9:7); scutellum smooth and slightly convex, posterior rim rounded (Fig. 5D); anterior scutellar pit large and deep, shorter than remaining scutellar disc, weakly crenulate at bottom, median keel present (Fig. 5D); mesopleuron predominantly smooth with deep crenulate line along posterior margin; metapleuron rugose and covered with dense long setae.

WINGs. Fore wing with costal, subcostal, basal, marginal, postmarginal, radial and stigmal veins tubular; medial vein pigmented; radial cell closed, as long as marginal vein and $3.0 \times$ its height (Fig. 5B).

LEGs. Fore and mid legs slender; hind tibiae gradually swollen.

Metasoma. Petiole slightly shorter than wide (5:6), strong costae dorsally; tergites completely smooth, with scattered setigerous punctures; base of second tergite with several short costae basally and short and shallow median furrow, extending $0.35 \times$ length of second tergite; sutures between tergites complete and deeply impressed.

CoLour. Body black; antennae yellowish brown basally gradually darkened towards apex; legs yellowish brown, except basal half of hind coxae blackish brown, hind femur partly brown, hind tibiae dark brown; tegulae yellowish brown; wings hyaline, covered with brown setae.

Measurements. Head length $0.44 \mathrm{~mm}$, width $0.82 \mathrm{~mm}$; mesosoma length $1.02 \mathrm{~mm}$, width $0.69 \mathrm{~mm}$; metasoma length $1.23 \mathrm{~mm}$; fore wing length $2.45 \mathrm{~mm}$; total body length $2.69 \mathrm{~mm}$.

Male (allotype)

Body length $1.93 \mathrm{~mm}$. Similar to female, but antenna uniformly brown to dark brown except scape and pedicel yellowish, basal half of all coxae dark brown (Fig. 5F); median furrow short and shallow, extending $0.4 \times$ length of second tergite; A4 excavate, curved (Fig. 5E); antennal segments in following proportions: $15: 6 ; 7: 5 ; 8: 4 ; 12: 5 ; 7: 8 ; 7: 5 ; 7: 5 ; 7: 5 ; 6: 5 ; 6: 5 ; 6: 5 ; 7: 5 ; 7: 5 ; 11: 5$ (Fig. 5E).

\section{Variation}

Body length $1.93-2.76 \mathrm{~mm}$ in both sexes; median furrow extending $0.35-0.4 \times$ length of second tergite in both sexes. 


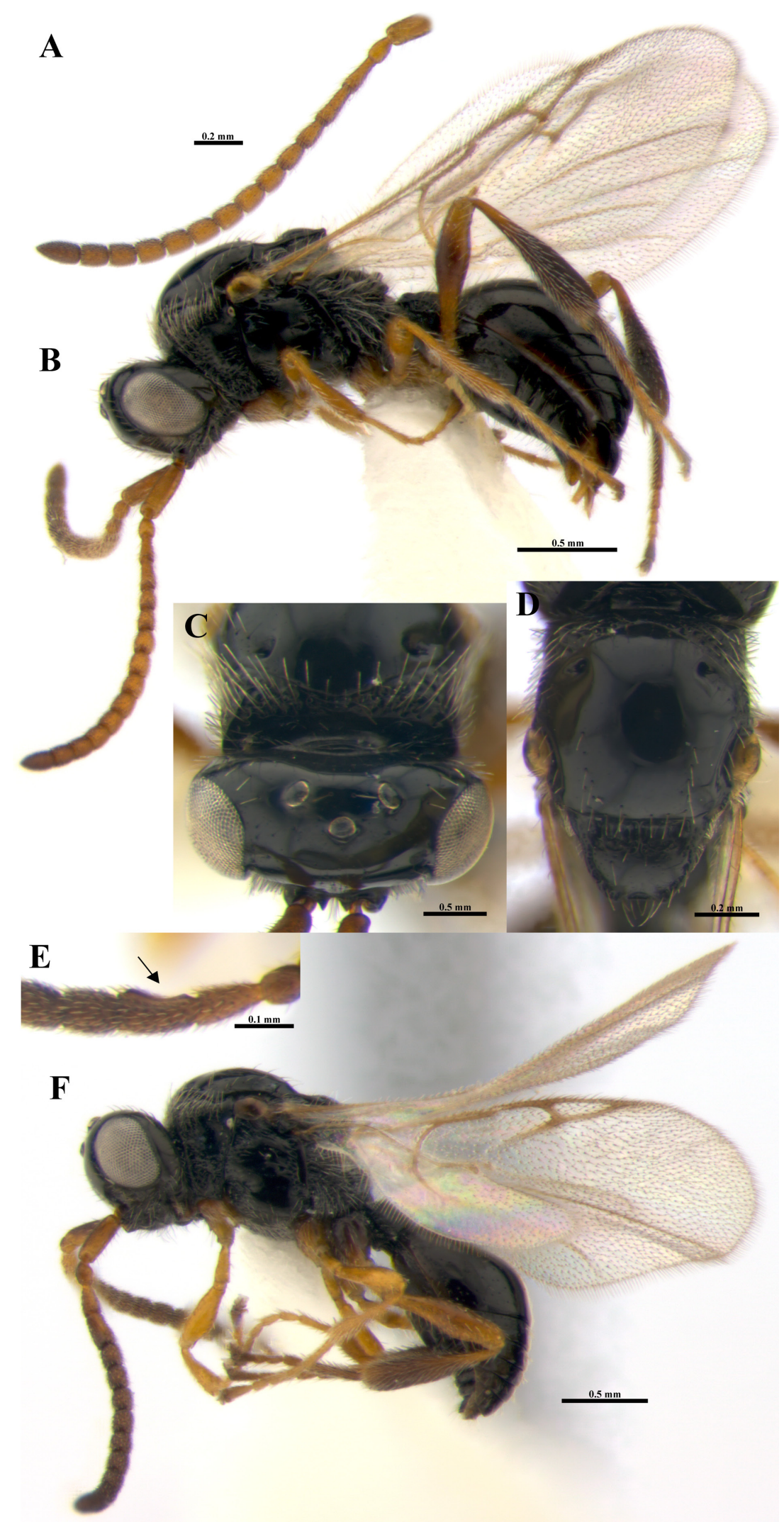

Fig. 5. Ismarus excavatus Kim \& Lee sp. nov. (A-D. Holotype, B. Habitus in lateral view. C. Head in dorsal view. D. Mesosoma in dorsal view. E. Antenna (A3-A5). F. Habitus in lateral view. 


\section{Host}

Unknown.

\section{Distribution}

China (Jilin), Japan (Aomori), South Korea.

Ismarus flavicornis (Thomson, 1858)

Figs $1 \mathrm{G}, \mathrm{I}, 2 \mathrm{C}$

Entomius flavicornis Thomson, 1858: 379.

Ismarus flavicornis - Marshall 1873: 8.

\section{Diagnosis}

Antenna uniformly bright yellow in female, pale brown to dark brown in male; mesopleuron with deep longitudinal wrinkles in both sexes, T2 with median furrow, at least to $2 / 3$ of segment in both sexes.

\section{Type material}

\section{Lectotype}

SWEDEN: + , "Sm" [Småland], "Bhn” [C.H. Boheman leg.], "NHRS-HEVA 000003606" (NHRS). Lectotype designated by Kolyada \& Chemyreva (2016).

\section{Additional material examined (35 우, $28 \hat{\jmath}$ ふै)}

BULGARIA: 1 đ̊, Burgas, above Nesebar, 13 Jun. 1969, B.H. Cogan, M.C. Cogan, R.I. Vane-Wright and R. Vane-Wright leg. (NHMUK010265065).

CANADA: 1 q, Québec, 16 km S of Louvicourt, 20 Jun. 1985, H. Goulet leg. (NHMUK010265068).

NORWAY: 1 +, Telemark, Kragerø, Knipenheia, 7 May-16 Jun. 2015, MT, F. Ødegaard leg. (NINA); 1 , Aust-Agder, Froland, Øyrekjerr, 5 Jun.-2 Jul. 2012, MT, A. Endrestøl leg. (NINA); 1 +, VestAgder, Kristiansand, Kjevikveien, 25 Jul.-3 Sep. 2009, MT, F. Ødegaard leg. (NINA).

SWEDEN: 1 ð̊, Blekinge, Sjöarp, 17 Jun. 1950, K.-J. Hedqvist leg. (NHMUK010265062); 1 +, Skåne, Höör Municipality, 13 Jun. 1938, D.M.S. Perkins and J.F. Perkins leg. (NHMUK010265054); 1 ๙, same collecting data, 16 Jun. 1938 (NHMUK010265066); 3 q , same collecting data, 17 Jun. 1938 (NHMUK010265053, NHMUK010265055, NHMUK010265060); 1 q, same collecting data, 26 Jun. 1938 (NHMUK010265059); 1 O̊, Västergötland, Kinnekulle, 12 Jun. 1975, K.-J. Hedqvist leg. (NHMUK010265063); 1 §ิ, Skåne, Kullaberg, 15 Jun. 1964, K.-J. Hedqvist leg. (NHMUK010265064); 1 Jె, Skåne, Ringsjön, 12 Jun. 1938, D.M.S. Perkins and J.F. Perkins leg. (NHMUK010265067); 2 우, Skåne, Röstanga, 6 Jul. 1938, D.M.S. Perkins and J.F.Perkins leg. (NHMUK010265052, NHMUK010265061); 1 + , Uppland, Vallentuna, 23 Jun. 1958, K.-J. Hedqvist leg. (NHMUK010265057); 1 , same collecting data, 26 Jun. 1959 (NHMUK010265056); 1 , same collecting data, 9 Jun. 2002 (NHMUK010265058).

UNITED KINGDOM: 1 đૈ, England, Beds, Dunton, TL237442, 24 Jun. 1973, V.H. Chambers leg. (NHMUK010265040); 1 $\hat{\partial}$, same collecting data, 1 Jul. 1973 (NHMUK010265036); $5 \hat{\partial} \widehat{\partial}$, same collecting data, 3 Jul. 1974 (NHMUK010265037, NHMUK010265038, NHMUK010265039, NHMUK010265042, NHMUK010265043); 1 §ิ, same collecting data, 9 Jul. 1974 (NHMUK010265041); 1 , England, Beds, Flitwick Moor, TL045350, Populus tremula, 15 Jun. 1963, V.H. Chambers leg. 
(NHMUK010264898); 1 §, same collecting data, under Betula, 27 Jun. 1978 (NHMUK010264902); $1 \widehat{\partial}$, same collecting data, Lonicera / Betula / Quercus, 19 Jun. 1980 (NHMUK010264904); 2 $\widehat{\partial}$, same collecting data, grass / Betula, 14 Jun. 1982 (NHMUK010264903, NHMUK010264905); 1 ठૈ, England, Beds, Flitwick Moor, TL045350, 7 Jul. 1984, V.H. Chambers leg. (NHMUK010265035); 1 9, England, Beds, Heath and Reach, 13 Jun. 1948, R.B. Benson leg. (NHMUK010264890); 1 ô, same collecting data, 13 Jun. 1948 (NHMUK010265046); 1 q, England, Beds, Kings Wood, SP920294, 12 Jun. 1949, V.H. Chambers leg. (NHMUK010264900); 4 우, same collecting data, Populus tremula, 1 Jul. 1951 (NHMUK010264894, NHMUK010264895, NHMUK010264896, NHMUK010264897); 1 \&, same

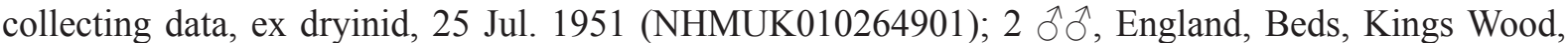
near Heath and Reach, 6 Jun. 1948, R.B. Benson leg. (NHMUK010265047, NHMUK010265051); 1 \%, England, Berks, Silwood Park, nr. Ascot, 11 Jun. 1994, D.G. Notton leg. (DNPC); 1 + , England, Bucks, Burnham Beeches, 13 Jun. 1976, Z. Bouček leg. (NHMUK010264893); 1 , England, Dorset, Studland Heath, 12-acre Wood, 22 Jun. 1934, G.J. Kerrich leg. (NHMUK010264892); 1 +, Scotland, Fife, Tentsmuir Nature Reserve, 16 Jul. 1977, J.S. Noyes, L. Rogers and T. Huddleston leg. (NHMUK010264899); 1 , England, Hants, Southampton, Jun. 1938, R.B. Benson leg. (NHMUK010264891); 3 q $q$, England, Herts, Brickett Wood, 13 Jun. 1943, R.B. Benson leg. (NHMUK010264882, NHMUK010264883, NHMUK010264887); 4 우, England, Herts, Brickett Wood, 17 Jun. 1936, R.B. Benson leg. (NHMUK010264884, NHMUK010264885, NHMUK010264886, NHMUK010264889); 1 +, England, Herts, Brickett Wood, 19 Jun. 1936, R.B. Benson leg. (NHMUK010264888); 1 đ̊, England, Kent, Ashford, 4 Jul. 1926, G.E.J. Nixon leg. (NHMUK010265044); 1 §, England, Northants, Spratton, Jun. 1975, I. Gauld and P. Gauld leg. (NHMUK010265045); 1 क, England, Surrey, Claygate, 19 Jun. 1953, D.M.S. Perkins and J.F. Perkins leg. (NHMUK010264881); 1 §ै, England, Surrey, Queen Mary Reservoir, nr Laleham, TQ061693, 28 May-4 Jun. 2011, R. Booth and A.Galsworthy leg. (NHMUK010265048); $2 \widehat{\jmath} \widehat{\jmath}$, same collecting data, 11 Jun. 2011 (NHMUK010265049, NHMUK010265050).

\section{Host}

Reared from Anteon flavicorne (Dalman) (Dryinidae) in France (Tussac \& Tussac 1991), Russia (Kozlov 1971), Switzerland (Wall 1967) and United Kingdom (Chambers 1955; Nixon 1957). In addition, we have excluded the reference of Waloff \& Jervis (1987), because they were not sure about the identity of the dryinid species (Anteon flavicorne or Anteon arcuatum) (Olmi, pers. comm.).

\section{Distribution}

Bulgaria (new record), Norway (new record), Austria (Masner 1976), Canada (Masner 1976), Czech Republic (Hellén 1964), Denmark (Johnson 2016), Estonia (Kolyada \& Chemyreva 2016), Finland (Hellén 1964), Germany (Wall 1967), Italy (Bin et al. 1995), Netherlands (Peeters 2015), Republic of Ireland (Nixon 1957; Stelfox 1966; O'Connor et al. 2004), Russia (European, Far East) (Kozlov 1971), Scotland (Wall 1967), Sweden (Kieffer 1916; Nixon 1957), Switzerland (Wall 1967), Ukraine (Kolyada \& Chemyreva 2016), United Kingdom (Nixon 1957; O'Connor et al. 2004), USA (Masner 1976).

\section{Ismarus grandis Alekseev, 1978}

Figs 1D, 2D

Ismarus grandis Alekseev, 1978: 1104.

\section{Diagnosis}

Antenna A4 as long as A1, as long as or slightly shorter than A3 in both sexes; male A4 slightly excavate; antennal segments distinctly elongate in both sexes; base of second tergite with long median furrow, at least to $2 / 3$ of segment in both sexes. 


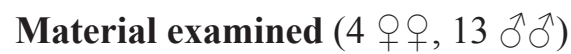

SOUTH KOREA: 1 q, Chungcheongbuk Province, Boeun-gun, Sokrisan-myeon, Mt. Sokri (Bench),

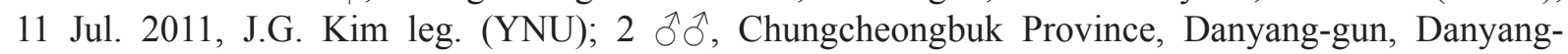

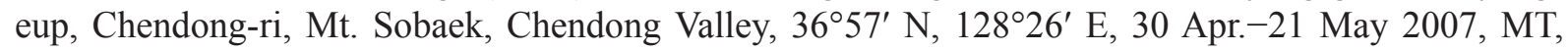
J.W. Lee (YNU); $1 \hat{\jmath}$, same collecting data, 21 May-17 Jun. 2007, MT, J.W. Lee leg. (YNU); 1 ${ }^{\lambda}$, Chungcheongbuk Province, Jecheon-si, Hamsu-myeon, Songgye 2-ri, 26 May 2007, J.W. Lee leg.

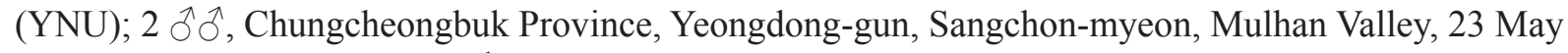
2002, J.W. Lee leg. (YNU); 1 §̂, Daejeon-si, Dong-gu, Daejeon-Univ., 12-27 May 2006, MT, J.W. Lee leg. (YNU); 1 đે, same collecting data, 16 May-5 Jun. 2006, MT, J.W. Lee leg. (YNU); 1 đ, Gangwon Province, Pyeonchang-gun, Yongpyeong-myeon, Nodong Valley, alt. $900 \mathrm{~m}, 37^{\circ} 42^{\prime} 08^{\prime \prime} \mathrm{N}, 128^{\circ} 28^{\prime} 89^{\prime \prime} \mathrm{E}$, 31 May-5 Jun. 2006, MT, in shade small stream, P. Tripotin leg. (CNCI); 1 §, Gyeongsangbuk Province, Cheongdo-gun, Unmun-myeon, Munsuseonwon, 35³8'32" N, 128 57'50" E, 23 Mar.-12 May 2013, MT, J.W. Lee leg. (YNU); 1 o, Gyeongsangbuk Province, Cheongdo-gun, Unmun-myeon, Simsimgyegok,

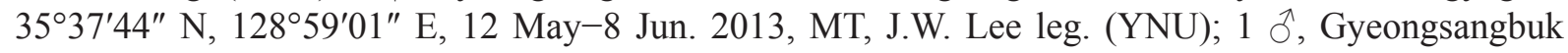

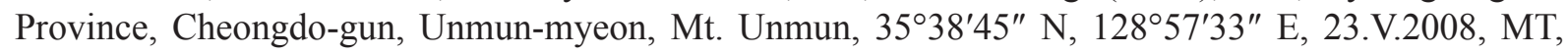
J.W. Lee leg. (YNU); 1 ${ }^{\lambda}$, Gyeongsangnam Province, Sancheong-gun, Mt. Jiri, Baengmudong, $35^{\circ} 20^{\prime} \mathrm{N}, 127^{\circ} 43^{\prime} \mathrm{E}, 12$ May 2002, J.W. Lee leg. (YNU); 1 ㅇ, Gyeongsangnam Province, Hamyang-

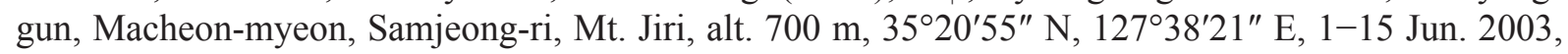
MT., big clearing on forest edge, P. Tripotin leg (CNCI).

JAPAN: 1 , Hokkaido, Jozankei, Naturem Village, alt. 380 m, 42 $55^{\prime} 48.7^{\prime \prime}$ N, 141 ${ }^{\circ} 09^{\prime} 10.1^{\prime \prime}$ E, 30 Jun. 2009, J.W. Lee leg. (YNU); 1 đ̂, Hokkaido, Kuriyama-cho, 7 Jun. 1996, M. Matsuda leg. (CNCI).

\section{Distribution}

Japan (new record), South Korea (new record), Russia (Far East) (Alekseev 1978).

Ismarus halidayi Förster, 1850

Figs $1 \mathrm{C}, \mathrm{H}, 2 \mathrm{E}$

? Entomia campanulata Herrich-Schäffer, 1840: 127 (nomen dubium).

Ismarus halidayi Förster, 1850: 285.

Ismarus longicornis Thomson, 1858: 378. Synonymized by Kolyada \& Chemyreva (2016).

Ismarus mongolicus Szabó, 1974: 23. Synonymized by Kolyada \& Chemyreva (2016).

\section{Diagnosis}

Antenna colour variable; POL as long as OOL in both sexes; A3 shorter than A4 in both sexes; radial cell as long as length of marginal vein in both sexes; base of second tergite with long median furrow, at least to half of segment in both sexes.

\section{Type material}

MONGOLIA: + , holotype of I. mongolicus, Central aimak, Tosgoni ovoo, 5-10 km N of Ulan-Baator, 1500-1700 m, Exp. Dr. Z. Kaszab, 19-20, 23-24 Jul. 1968 (HNHM, Typ. No. 2622, Mus. Budapest) holotype by original designation.

SWEDEN: + , lectotype of I. longicornis, "Sbg" [Sövdeborg], "Rh" [Carl David Emmanuel Roth leg.], "NHRS-HEVA 000003605" (NHRS) lectotype designated by Kolyada \& Chemyreva (2016).

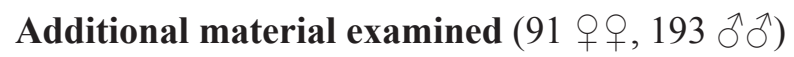

CANADA: 1 , Newfoundland, South Branch, Jul. 1974, MT, Heinrich leg. (NHMUK010265151). 
REPUBLIC OF IRELAND: 1 + , Co. Wicklow, Buckroney, 15 Jun. 1949, M.W.R. de V. Graham leg. (NHMUK010265375).

GERMANY: 1 , , North Rhine-Westphalia, Leverkusen, Bergisch-Neukirchen, River Wupper, reared from flood debris, 3 Feb. 2002, M. Boness leg. (DNPC); 1 đ̆, same collecting data, 2 Jan. 1982 (DNPC).

NORWAY: 1 , Vestfold, Stokke, Melsomvik, 19 Jun. 2012, A. Staverløkk leg. (NINA); 1 , Vestfold, Sandefjord, 20 Jun. 2012, A. Staverløkk leg. (NINA); 1 đે, Vestfold, Horten, Løvøya, 27 May-16 Jun. 2014, MT, A. Staverløkk leg. (NINA); 1 đ, Vestfold, Stokke, Feen, 19 Jun. 2012, A. Staverløkk leg. (NINA); 5 우오, Aust-Agder, Froland, Øyrekjerr, 2 July-14 Aug. 2012, MT, A.Endrestøl (NINA); 1 ㅇ, same collecting data, 5 Jun.-2 Jul. 2015; 1 §, 1 \&, Sogn og Fjordane, Sogndal, Fiksneset, 5 Jul.-10 Aug. 2011, MT, F. Ødegaard leg. (NINA); 1 +, Sør-Trøndelag, Oppdal, Helvetesdalen, 29 Jun.-17 Jul. 2014, MT, O. Hanssen leg. (NINA); 1 §, Sør-Trøndelag, Trondheim, Jonsvannet, Tangen, 31 May-30 Jun. 2015,

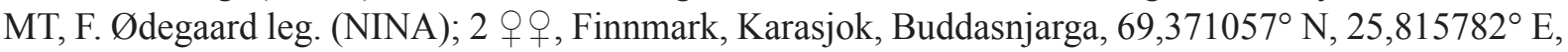
26 Jun.- 12 Jul. 2016, MT, F. Ødegaard leg. (NINA); 1 ㅇ, same collecting data, 12 Jul.-11 Aug. 2016.

SOUTH KOREA: 1 $\widehat{\jmath}$, Chungcheongbuk Province, Chungju-si, Suanbo-myeon, Samun-ri, Mt.

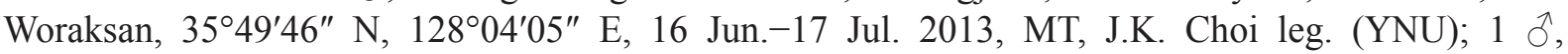
Chungcheongbuk Province, Boeun-gun, Mt. Songni National Park, Beopjusamaepyoso, 36 32'06" N, 127 49'40" E, 12 May-21 Jun. 2007, MT, J.W. Lee leg. (YNU); $1 \overbrace{}^{\lambda}$, Chungcheongbuk Province, Boeun-gun Mt. Songnisan, Beopjusa, 36³2'06" N, 12749'40" E, 5 May-31 Aug. 2011, MT, J.C. Jung

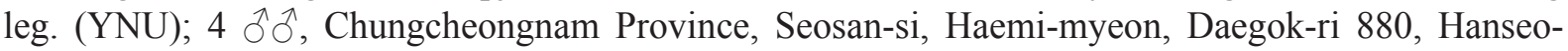
Univ., 3641'30" N, 126³4'50" E, 14 May-11 Jun. 2009, MT, J.W. Lee leg. (YNU); 1 ô, Daejeon-si, Dong-gu, Daejeon-Univ., 13-27 May 2006, MT, J.W. Lee leg. (YNU); 9 $\widehat{\jmath}, 1$, , same collecting data, 16 May-5 Jun. 2006, MT, J.W. Lee leg. (YNU); $2 \hat{\jmath} \hat{\jmath}, 1$, same collecting data, 12-27 May 2007, MT, J.W. Lee leg. (YNU); 1 đ, 6 우, Daejeon-si, Seo-gu, Polpyeong-dong, Wolpyeong Park, 36 20'30" N, $127^{\circ} 21^{\prime} 30^{\prime \prime}$ E, 12 May-24 Jun. 2008, MT, J.W. Lee leg. (YNU); $3{ }^{\lambda}{ }^{\lambda}$, Daejon-si, Wadong, 36 $24^{\circ} 02^{\prime \prime} \mathrm{N}$, $127^{\circ} 25^{\prime} 98^{\prime \prime}$ E, 6-28 May 2006, MT, edge of wild rose patch, P. Tripotin leg. (CNCI); 10 § $0^{\top}, 3$ 우우, same collecting data, 28 May-19 Jun. 2006, MT, edge of wild rose patch, P. Tripotin leg. (CNCI); 1 q,

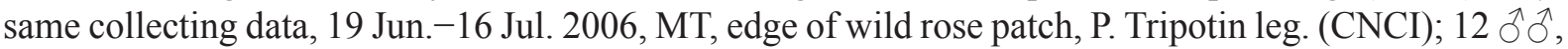
1 ㅇ, Gangwon Province, Chuncheon-si, Nam-myeon, Hudong-li, 25 May-14 Jun. 2003, MT, semishade, forest edge, P. Tripotin leg. (CNCI); 1, 2 우, Gangwon Province, Chuncheon, Nam-myeon,

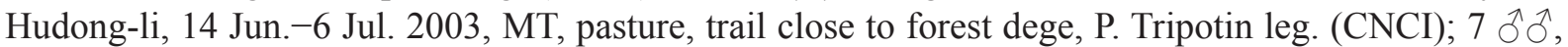

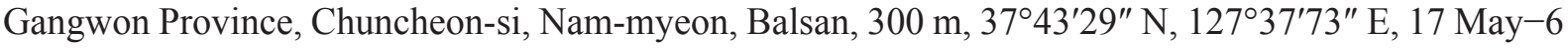
Jun. 2006, MT, forest, P. Tripotin leg. (CNCI); 2 $\widehat{\jmath}$, same collecting data, 26 Jun.-30 Jul. 2006, MT, forest, P. Tripotin leg. (CNCI); 1 $\hat{\lambda}$, Gangwon Province, Inje-gun, Girin-myeon, Mt. Bangtaesan,

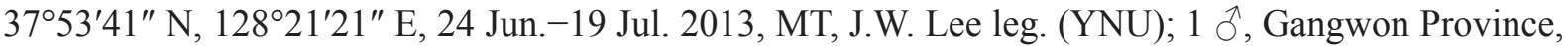
Gwanggwon Prov. Environment Park, Bukbang, Hongchoen, 3545'15.6" N, 127 51'1.7" E, 30 May-15 Jun. 2012, MT, S.J. Jang leg. (YNU); 1 §, Gangwon Province, Hoengseong-gun, Gapcheon-myeon,

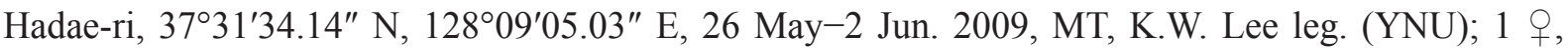

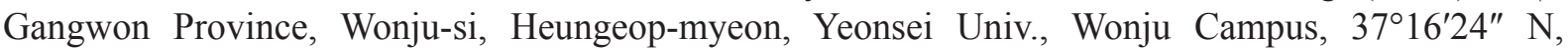

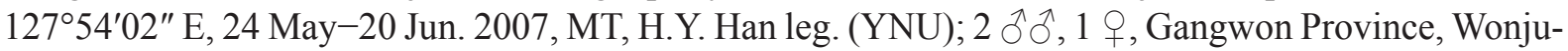

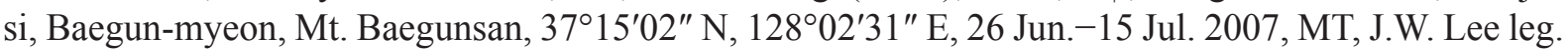

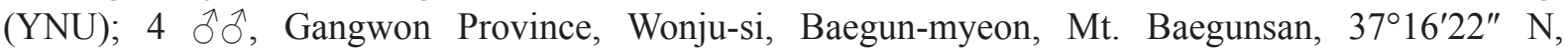
127055'65" E, 19 Jun.-5 Jul. 2011, MT, J.W. Lee leg. (YNU); 1 §, Gangwon Province, Wonju-si,

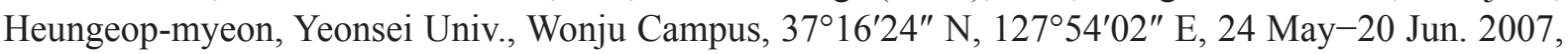
MT, H.Y. Han leg. (YNU); 1 q, Gangwon Province, Wonju-si, Heungeop-myeon, Maeji-ri, Yonsei

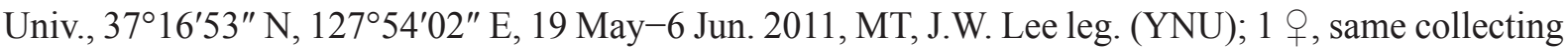
data, 29 May-5 Jul. 2015, MT, H.Y. Han leg. (YNU); 2 $\widehat{\jmath}$, Gangwon Province, Wonju-si, Socho-

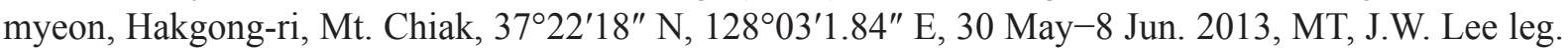


(YNU); 1 , , same collecting data, 9-20 Jun. 2013, MT, J.W. Lee leg. (YNU); 1 + , Gyeonggi Province, Anvang-si, Manan-gun, Kwanag Arb., 9-24 Jun. 2007, MT, J.O. Lim leg. (YNU); 1 ô, 1 क, Gyeonggi

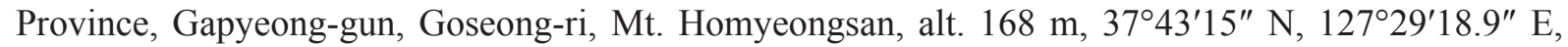
1 May-26 Jun. 2009, MT, J.O. Lim leg. (YNU); 1 क, same collecting data, 26 Jun.-16 Jul. 2009, MT,

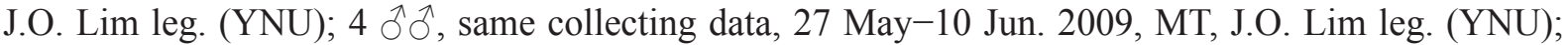

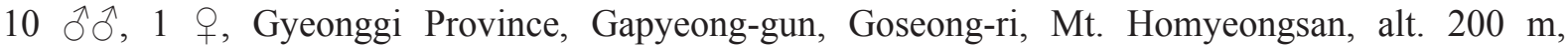

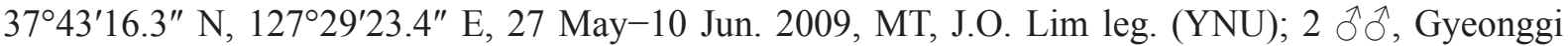
Province, Gapyeong-gun, Cheongpyeong-myeon, Goseong-ri, Mt. Homyeongsan, alt. $220 \mathrm{~m}$,

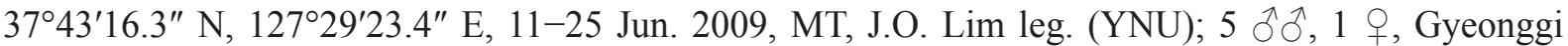
Province, Gwangju-si, Docheog-myeon, Mt. Taehwasan, alt. 219 m, 37 $18^{\prime} 05^{\prime \prime} \mathrm{N}, 127^{\circ} 19^{\prime} 01^{\prime \prime} \mathrm{E}$, 8 May-7 Jun. 2007, MT, J.O. Lim leg. (YNU); 1 §,, same collecting data, 25 May-8 Jun. 2007, MT, J.O. Lim leg. (YNU); 5 $\widehat{\partial}, 2$ 9 ㅇ, same collecting data, 9-24 Jun. 2007, MT, J.O. Lim leg. (YNU); 8 $\widehat{\partial}$, 7 우, Gyeonggi Province, Namyangju-si, Choan-myeon, Songchon-ri, Mt. Ungilsan, alt. 134 m, $37^{\circ} 34^{\prime} 43^{\prime \prime}$ N, $127^{\circ} 18^{\prime} 37^{\prime \prime}$ E. 27 May-10 Jun. 2009, MT, J.O. Lim leg. (YNU); 2 오, same collecting

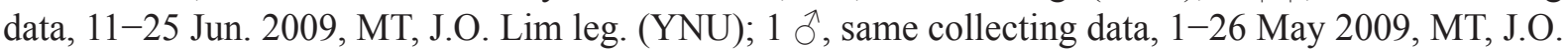
Lim leg. (YNU); 1 $\widehat{\text {, }}$, Gyeonggi Province, Suwon-si, Kwonseon-gu, Seodon-dong, Suwon Arb., alt. 42 m, 37 15'38.3" N, 126 59'01.1" E, 20 May-10 Jun. 2009, MT, J.O. Lim leg. (YNU); 1 ð, Gyeonggi Province, Yangpyeong-gun, Yongmun-myeon, Yeonsu-ri, Mt. Yongmunsan, 37³1'49.5" N, 127³4'18.8" E, 11-25 Jun. 2009, MT, J.O. Lim leg. (YNU); 1 §, Gyeongsangbuk Province, Cheongdo-

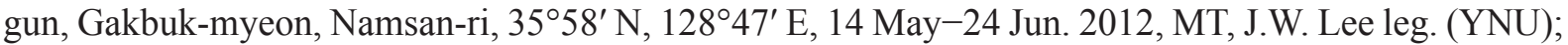

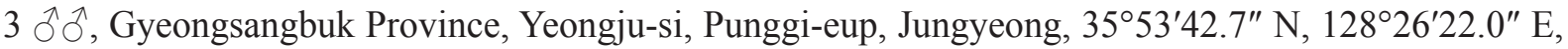
21 May-3 Jun. 2009, MT, C.J. Kim leg. (YNU); 5 đิ đે, same collecting data, 3-12 Jun. 2009, MT, C.J. Kim leg. (YNU); 5 $\widehat{\partial}$, same collecting data, 12-22 Jun. 2009, MT, C.J. Kim leg. (YNU); 1 q, same collecting data, 22 Jun.-3 Jul. 2009, MT, C.J. Kim leg. (YNU); 2 우, Gyeongsangnam Province, Hamyang-gun, Macheon-myeon, Samjeong-ri, Mt. Jirisan, alt. 700 m, 35 $20^{\prime} 55^{\prime \prime}$ N, 127 $38^{\prime} 21^{\prime \prime}$ E,

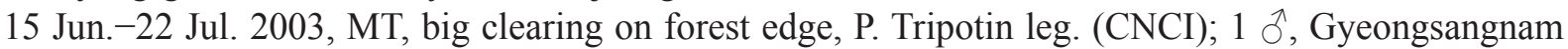
Province, Samcheong-gun, Samjang-myeon, Yupyeon-ri, Wangdeungjae, Mt. Jiri National Park,

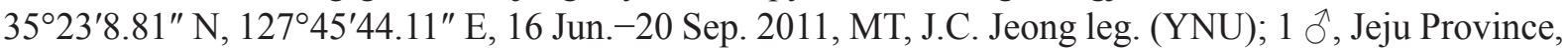

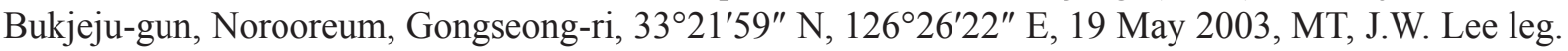
(YNU); 1 , Jeollanam Province, Jangeong-gun, Bukha-myeon, Mt. Naejangsan, Sajabong, $36^{\circ} 24^{\prime} 14.01^{\prime \prime}$ N, 126 52'12.09" E, 21 Jun. 2005, MT, K.B. Kim leg. (YNU); 1 đ, Jeollanam Province, Jangeong-gun, Bukha-myeon, Mt. Naejangsan, Baegyangsa, 35 $26^{\prime} 22.8^{\prime \prime} \mathrm{N}, 126^{\circ} 53^{\prime} 00^{\prime \prime}$ E, 18 Apr.-30 May 2008, MT, J.W. Lee leg. (YNU); 1 q, same collecting data, 30 May-5 Aug. 2008, MT, J.W. Lee leg. (YNU); 1 , Jeollabuk Province, Jeongeup-si, Ibam-myeon, Deungcheon-ri, Wetland $35^{\circ} 28^{\prime} 35.95^{\prime \prime}$ N, 126 47'59.17" E, 21 Jun. 2005, MT, M.B. Yun leg. (YNU); 1 Oૈ, Jeollabuk Province, Jeongeup-si, Naejang-dong, Mt. Naejangsan, Wonjeogam, 35²9'36" N, 126 53'37" E, 13-29 May 2007, MT, J.W. Lee leg. (YNU); 2 ổ, same collecting data, 20 Jun. 2005, MT, J.W. Lee leg. (YNU); 1 , Jeollabuk Province, Jeongeup-si, Singjeong-dong, Mt. Naejangsan, Namchanggol, $35^{\circ} 27^{\prime} 48^{\prime \prime}$ N, 126 50'18" E, 30 May-5 Jul 2008, MT, J.W. Lee leg. (YNU); 8 $\jmath^{\circ}, 3$ 우, Jeollabuk Province, Jeongeup-si, Yongsan-dong, 35¹9'8.97" N, 12653'11.74" E, 19 May-19 Jun. 2004, MT, M.K. Yun leg.

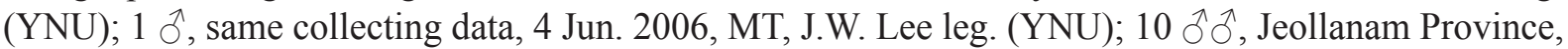

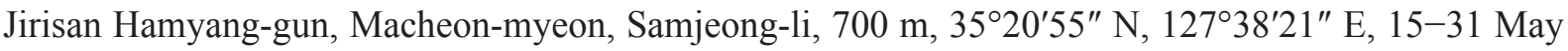
2003, MT, forest clearing, stream, P. Tripotin leg. (CNCI); 1 ते, Jeollanam Province, Jirisan, Hamyang-

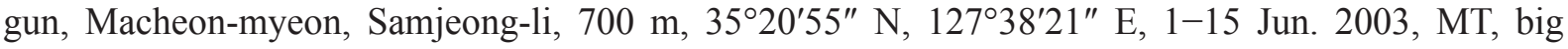
clearing, forest edge, P. Tripotin leg. (CNCI); 1 ${ }^{\Uparrow}$, Jeollanam Province, Jirisan, Hamyang-gun, Macheon-

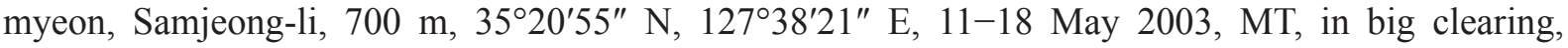

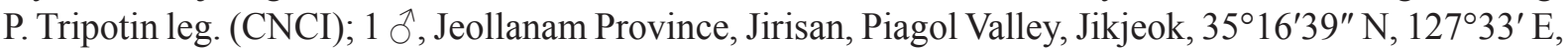
1-15 Jun. 2003, MT, in shade in small clearing in forest, C. Young leg. (CNCI); 1 o, 1 क , Jeollanam Province, Yeongam-gun, Hoemun-ri, Daedongjae, 22 May-31 Jul. 2010, MT, J.K. Kim leg. (YNU);

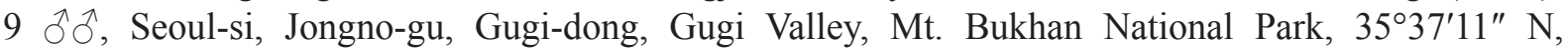


126 57'42" E, 5 Jun.-22 Jul. 2010, MT, J.C. Jeong leg. (YNU); 1 , Gyeongsangbuk Province, Mungyeong-si, Gaeun-eup, Wonjang-ri, Mt. Songnisan, Beorimgijae, 36 $40^{\prime} 59^{\prime \prime}$ N, $127^{\circ} 57^{\prime} 07^{\prime \prime}$ E, 21 May-15 Jun. 2013, J.K. Choi leg. (YNU).

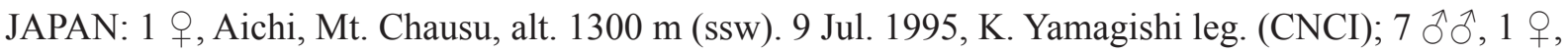
Hokkaido, Sapporo, 24-29 Jul. 1988, MT, K. Maeto leg. (CNCI); 1 §ૈ, Hokkaido, Sapporo, Hitsujgaoka,

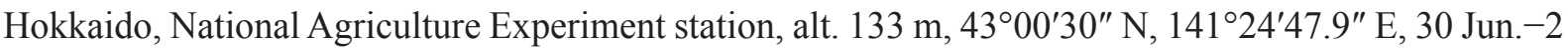
Jul. 2008, MT, J.W. Lee leg. (YNU); 1 ㅇ, Nishioka-Park, Toyohira-ku, Sapporo-shi, Hokkaido, alt. 140 m, 42 59'19.3" N, 141 $22^{\prime} 46.9^{\prime \prime}$ E, 27 Jul. 2013, MT, J.W. Lee leg. (YNU).

RUSSIA: 1 ○, Primorsky-Krai, Vladivostok, 8-15 Jun. 2008, MT, J.W. Lee leg. (YNU); 1 , , Primorsky-

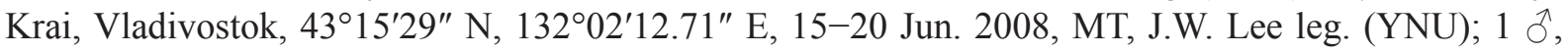
Primorsky-Krai, Vladivostok, 20 Jun.-5 Jul. 2008, MT, J.W. Lee leg. (YNU).

SWEDEN: 1 đ̂, Västerbotten, Hällnäs, 3 Jul. 1960, K.-J. Hedqvist leg. (NHMUK010265149);

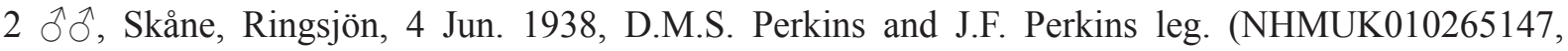
NHMUK010265148); 1 q, same collecting data, 24 Jun. 1938 (NHMUK010265143); 1 , same collecting data, 27 Jun. 1938 (NHMUK010265144); 1 \&, Uppland, Vallentuna, Jun. 1957, K.-J. Hedqvist leg. (NHMUK010265146); 1 , same collecting data, 31 May 2007 (NHMUK010265145).

UNITED KINGDOM: 1 क, England, Beds, Flitwick Moor, TL045350, Betula, 25 Jun. 1960, V.H. Chambers leg. (NHMUK010265076); 1 ô, same collecting data (NHMUK010265092); 1 \%, same collecting data, Lonicera / Betula / Quercus, 19 Jun. 1980 (NHMUK010265078); 1 ô, same collecting data, Lonicera, 29 Jun. 1981 (NHMUK010265091); 1 , England, Beds, Flitwick Moor, TL045350, 17 Jul. 1981, V.H. Chambers leg. (NHMUK010265080); 1 ㅇ, England, Beds, Kings Wood, SP920294, Betula, 11 Jul. 1951, V.H. Chambers leg. (NHMUK010265074); 1 §, same collecting data, Betula, 21 Jun. 1953 (NHMUK010265087); 1 \&, same collecting data, Betula, 26 Jun. 1953 (NHMUK010265075); 1 q, same collecting data, Betula, 3 Aug. 1953 (NHMUK010265084); 1 ô, same collecting data, bred, host collected 11 Jul. 1953, wasp emerged 16 Jun. 1954, ex Anteon sp. on Oncopsis sp. on Betula sp. (NHMUK010265089); 1 Oे, England, Beds, path to Sharpenhoe Clappers, TL063298, Alnus glutinosus, 12 Jul. 1951, V.H. Chambers leg. (NHMUK010265088); 1 q, England, Beds, Sutton Fen, TL202475, Betula, 18 Jul. 1978, V.H. Chambers leg. (NHMUK010265079); 1 + , same collecting data, fern, 2 Jul. 1980 (NHMUK010265077); 1 \&, England, Bucks, Soulsbury, Rammamere Heath, 4-17 Jul. 1944, R.B. Benson leg. (NHMUK010265072); 1 q, England, Bucks, Wootton Underwood, 4 Jul. 1957, R.B. Benson leg. (NHMUK010265081); 1 ㅇ, England, Cambs, Chippenham Fen, TL650693, carr / reedbed, ?-6 Jul. 1983, MT, J. Field leg. (DNPC); 1 , England, Ches, Abbots Moss, SJ596680, by stream, 1-19 Jun. 1990, MT, D.G. Notton leg. (DNPC); 1 ふૈ, same collecting data, 19 Jun.-10 Jul. 1990 (DNPC); 1 을 England, Devon, Dartmoor, Lustleigh, Jun. 1934, R.C.L. Perkins leg. (NHMUK010265071); 1 ô, Scotland, Glasgow, Clober nr Milngavie, pre-1910, P. Cameron leg. (NHMUK010265139); 1 ○, England, Hants, Farley, 12 Jun. 1938, R.B. Benson leg. (NHMUK010265095); 1 +, England, Herts, Tring, 16 Jul. 1942, R.B. Benson leg. (NHMUK010265073); 1 \%, Scotland, Highland, Aviemore, 30 Jun. 1934, R.B. Benson leg. and J.E. Benson leg. (NHMUK010265138); 1 đ, same collecting data, 4 Jun. 1952, R.B. Benson leg. (NHMUK010265142); 1 స, Scotland, Highland, Bonar, pre-1910, P. Cameron leg. (NHMUK010265086); 1 $\hat{\jmath}$, same collecting data, pre-1909 (NHMUK010265141); 1 , Scotland, Highland, Kinlochewe, 1-8 Jun. 1961, R.B. Benson leg. (NHMUK010265137); 1 ;, England, Lancs, Freshfield, 28 Jul. 1961, M.W.R. de V. Graham leg. (NHMUK010265082); 1 o, England, Norfolk, Santon Downham, TL818883, MT, ? -6 Jul. 1985, J. Field leg. (DNPC); 2 qq Scotland, Perth and Kinross, Ballinluig, 20 Jul. 1977, J.S. Noyes, L. Rogers and T. Huddleston leg. (NHMUK010265097, NHMUK010265136); 1 ग̃, England, Suffolk, Brandon, 23-30 May 1945, R.B. Benson leg. (NHMUK010265096); 1 q, England, Surrey, Horsley, 12 Jun. 1957, J.F. Perkins 
leg. (NHMUK010265083); 1 đૈ, England, Surrey, Oxshot, 25 Jun. 1937, G.E.J. Nixon leg. (NHMUK010265094); 2 §ð, England, 12 Jun. 1966, V.H. Chambers leg. (NHMUK010265085, NHMUK010265090).

\section{Variation}

Body length 1.93-3.79 $\mathrm{mm}$ in both sexes; antenna colour variable in both sexes: totally dark brown or brown, dark brown or brown except segments 1 or 1-2 to 1-10 yellowish; anterior scutellar pit with median keel present or absent, weakly crenulate or completely smooth at bottom in both sexes; hind leg colour variable in both sexes: femur yellow to brown, tibia and tarsus yellow to dark brown; median furrow extending $0.5-0.9 \times$ length of second tergite in both sexes.

\section{Distribution}

Japan (new record), South Korea (new record), Azerbaijan (Kolyada \& Chemyreva 2016), Bulgaria (Petrov 1990), Canada (Masner 1976), China (Ningxia, Sichuan, Guizhou, Yunnan, Tibet)(Liu et al. 2011), Czech Republic (Hellén 1964), Denmark (Johnson 2016), Finland (Hellén 1964), Georgia (Kolyada \& Chemyreva 2016), Germany (Kieffer 1916), Hungary (Kieffer 1916; Peeters 2015), Mongolia (Szabó 1974), Netherlands (Peeters 2015), Norway (http://www.biodiversity.no/Pages/135494), Republic of Ireland (Stelfox 1966; O'Connor et al. 2004), Russia (European, Far East, Siberia) (Kolyada \& Chemyreva 2016), Scotland (Nixon 1957), Sweden (Nixon 1957), United Kingdom (Kieffer 1916; Nixon 1957, Notton 1996; O’Connor et al. 2004), USA (Masner 1976).

\section{Host}

Reared from Anteon jurineanum Latreille, 1809 (Chambers 1955, as A. brevicorne; Olmi 2000) and A. infectum (Haliday) (Dryinidae) (Chambers 1955, 1981), both in United Kingdom. However, Chambers (1981: as I. halidayi), but not Chambers (1955), which is in fact I. similis sp. nov., see below.

\section{Notes}

Kolyada \& Chemyreva (2016) incorrectly state that Entomia campanulata is a nomen oblitum, however no action has been taken under Article 23.9.2 (ICZN 1999) to reverse the precedence with respect to I. halidayi so it cannot be a nomen oblitum. We follow Masner (1976) who doubtfully included in it synonymy with Ismarus halidayi and we consider that the interpretation of the species is doubtful and so the synonymy is also doubtful, and it is therefore a nomen dubium, i.e., although it is the older name, it is not certainly the valid name for the species referred to here as I. halidayi.

\section{Ismarus multiporus Kolyada \& Chemyreva, 2016}

Fig. $2 \mathrm{~F}$

Ismarus multiporus Kolyada \& Chemyreva, 2016: 12.

\section{Diagnosis}

Notauli with 5-8 pits in both sexes; radial cell as long as marginal vein in both sexes; posterior half of S6 yellow in both sexes.

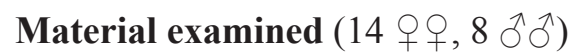

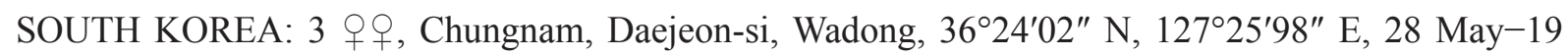
Jun. 2006, MT for edge wild rose patch, P. Tripotin leg. (CNCI); 1 q, same collecting data, 19 Jun. -16 Jul. 2006, MT for edge wild rose patch, P. Tripotin leg. (CNCI); 1 ㅇ, Kangwon, Chuncheon, Nammyeon, Hudong-li, 25 May-14 Jun. 2003, MT for pastured area, trail close to forest edge, P. Tripotin 
leg. (CNCI); 2 qq , same collecting data, 14 Jun. -6 Jul. 2003, MT for pastured area, trail close to forest edge, P. Tripotin leg. (CNCI); 1 \%, Gangwon, Chuncheon-si, Nam-myeon, Udong-li, 26 Jun.-30 Jul. 2006, MT for forest edge, P. Tripotin leg. (CNCI); 2 우, Jirisan, Hamyang-gun, Macheon-myeon, Samjeong-li, $700 \mathrm{~m}, 35^{\circ} 20^{\prime} 55^{\prime \prime} \mathrm{N}, 127^{\circ} 38^{\prime} 21^{\prime \prime}$ E, 15-22 Jun. 2003, MT for big clearing on embankment, P. Tripotin leg. (CNCI); 1 ô, Daejeon, Dong-gu, Daejeon Univ., 16 May-5 Jun. 2006, MT, J.W. Lee leg. (YNU); 1 $\lambda$, Jeollabuk Province, Jeongeup-si, Naejang-dong, Mt. Naejangsan, Wonjeogam,

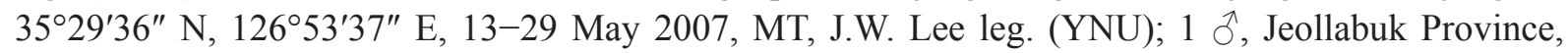
Jeongeup-si, Naejang-dong, Mt. Naejangsan, Geumseonggyegok, Yonggul, 35²9'15" N, 126 53'34" E, 13-29 May 2007, MT, J.W. Lee leg. (YNU); 1 O, Jeollabuk Province, Jeongeup-si, Yongsan-dong, 35¹9'8.97" N, 12653'11.74" E, 19 May-19 Jun. 2004, MT, M.K. Yun leg. (YNU); 1 ô, Jeollanam

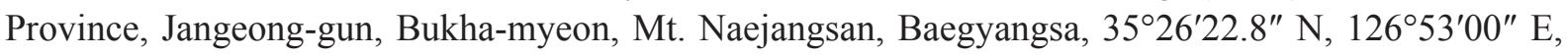

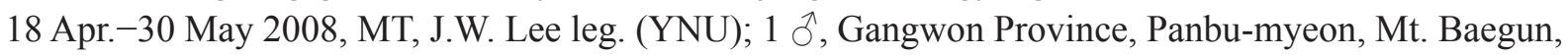
$37^{\circ} 15^{\prime} 30^{\prime \prime}$ N, 12758'55" E, 26 Jun.-15 Jul. 2011, MT, J.W. Lee leg. (YNU); 1 §, Chungcheongnam Province, Gongju-si, Banpo-myeon, Donghae-si, Mt. Gyeryong, 36 21'37" N, 127 $14^{\prime} 23^{\prime \prime}$ E, 15 Mar.-20 Oct. 2012, MT, J.C. Jeong leg. (YNU).

JAPAN: 1 đ̃, 1 †, Hokkaido, Sapporo, 24-29 Jul. 1988, MT, K. Maeto leg. (CNCI); 1 \&, Hokkaido, Sapporo, sweep For. Res. Station, 28 Jul. 1989, M.J. Sharkey leg. (CNCI); 1 , , Aichi, Mt. Chausu, 1300 m (ssw), 9 Jul. 1995, K. Yamagishi leg. (CNCI); 1 +, Aichi, 900 m, Shitara, Uradani (beech forest), 23 Jun. -3 Jul. 1994, MT, K. Yamagishi leg. (CNCI).

\section{Variation}

Two male specimens from Daejeon, South Korea and Hokkaido, Japan have body the colour chestnut brown to dark brown, not blackish. Notauli with 5-8 pits in both sexes; median furrow extending $0.7-0.9 \times$ length of second tergite in both sexes.

\section{Distribution}

South Korea (new record), Japan (new record), Russia (Far East) (Kolyada \& Chemyreva 2016).

Ismarus rugulosus Förster, 1850

Figs $1 \mathrm{E}, 2 \mathrm{G}$

Ismarus rugulosus Förster, 1850: 284.

Ismarus rugulosus - Ashmead 1893: 380

Entomius rugulosus - Thomson 1858: 379. Generic transfer.

\section{Diagnosis}

Antenna uniformly brown to dark brown in both sexes; posterior part of scutellum coriaceous in both sexes; mesopleuron coriaceous-rugulose in both sexes; metasoma deeply scaly-reticulate in both sexes.

Material examined $(80 \stackrel{+}{\circ, 1}$ ふ)

AUSTRIA: 3 qq , Tyrol, Neustift im Stubaital, 1050 m, 7 Jul. 1995, MT, C.J. Zwakhals leg. (NHMUK010265182, NHMUK010265183, NHMUK010265184); 1 q, Tyrol, Virgen, 1450 m, MT, 31 Jul. 1992, C.J. Zwakhals leg. (DNPC).

CANADA: 1 q, Ontario, Ottawa, pan trap, 28 Jun. 1987, H. Goulet leg. (NHMUK010265188). 
GERMANY: 2 + , Baden-Württemberg, Kraichgau region, NE of Karlsruhe, suction trap, three year old fallow, mown, 29 Jun. 1990, H.-J.Greiler (DNPC); 1 ô, North Rhine-Westphalia, Leverkusen, reared from flood debris, 24 Feb. 1970, M. Boness leg. (DNPC); 1 q, pre-1859, J.F. Ruthe leg. (NHMUK010265186).

NORWAY: 1 , Østfold, Hvaler, Ørekroken, 14 Jun.-18 Jul. 2007, MT, F. Ødegaard leg. (NINA); 1 , Østfold, Halden, Fredriksten, 27 Jul.-21 Aug. 2010, MT, F. Ødegaard leg. (NINA); 1 +, Buskerud, Kongsberg, Laugerudmoen, 5 Jul.-8 Aug. 2014, MT, F. Ødegaard leg. (NINA); 2 + + , Akershus, AurskogHøland, Svarttjennhøgda, 10 Aug.-14 Sep. 2015, MT, A. Staverløkk leg. (NINA); 1 , Buskerud, Lier, Toverud, 24 Jul.-1 Oct. 2015, MT, F. Ødegaard leg. (NINA); 1 \&, Buskerud, Øvre Eiker, Hokksund, Nordre Haga, 5-23 Jul. 2016, MT, F. Ødegaard leg. (NINA); 1 ㅇ, Hedmark, Elverum, Starmoen, 13 Jun.-19 Jul. 2007, MT, F. Ødegaard leg. (NINA); 2 우, Hedmark, Eidskog, Magnor, Vanga, 10 Aug.14 Sep. 2015, MT, A. Staverløkk leg. (NINA); 1 \&, Vestfold, Horten, Borrevann, 1 Jul.-2 Aug. 2015, MT, A. Staverløkk leg. (NINA); 1 क, Telemark, Siljan, Brenndalskarven, 3 Jul.-8 Aug. 2015, MT, F. Ødegaard leg. (NINA); 1 \&, Aust-Agder, Froland, Øyrekjerr, 5 Jun.-2 Jul. 2012, MT, A. Endrestøl leg. (NINA); 1 q, Aust-Agder, Grimstad, Sandkleiv, 7 Aug.-19 Sep. 2015, MT, F. Ødegaard leg. (NINA); 2 우, Vest-Agder, Birkenes, Vassbotn, 25 Jun.-19 Jul. 2016, MT, A. Staverløkk leg. (NINA); 1 q, Møre og Romsdal, Norddal, Løberget, 22 Jul.-24 Aug. 2015, MT, O. Hanssen leg. (NINA); 1 \%, SørTrøndelag, Trondheim, Jonsvannet, Tangen, 12 Jul.-9 Sep. 2011, MT, F. Ødegaard leg. (NINA); 2 우, Sør-Trøndelag, Midtre-Gauldal, Staverløkja, 26 Aug.-1 Sep. 2012, MT, A. Staverøkk leg. NINA); 2 웅, Sør-Trøndelag, Midtre-Gauldal, Svintjønna, 22 Aug. 2016, MT, A. Staverøkk leg. (NINA); 1 ㅇ, Finnmark, Karasjok, Heastanjarga, 69.402084 N, 25.7515 E, 12 Jul. 2016, MT, F. Ødegaard leg. (NINA).

REPUBLIC OF IRELAND: 1 q, Co. Dublin, Glenasmole, 2 Jul. 1941, A.W. Stelfox leg. NHMUK010265374).

SWEDEN: 1 q, Skåne, Åhus, 31 Jul. 1970, K.-J. Hedqvist leg. (NHMUK010265177); 1 q, same collecting data, 21 Jun. 1989 (NHMUK010265179); 1 \&, Gotlands, Gotska Sandön, 28 Jun. 1953, K.J. Hedqvist leg. (NHMUK010265180); 1 , Bohuslän, Hålta, PK67602075, 100 m, swept, grassland with horses, by Quercus woodland, 21 Jun. 1992, M. Söderlund leg. (NHMUK010265181); 1 +, Skåne, Kivik, 16 Jul. 1938, D.M.S. Perkins and J.F. Perkins leg. (NHMUK010265176); 1 \%, same collecting data, 20 Jul. 1938 (NHMUK010265175); 1 ㅇ, Skåne, Trolle Ljunby, 29 Jul. 1974, K.-J. Hedqvist leg. (NHMUK010265178).

UNITED KINGDOM: 1 + , England, Beds, Eaton Bray Down, s.w.slope, 16 Jul. 1967, V.H. Chambers leg. (NHMUK010265158); 1 + , England, Berks, Silwood Park, Ashurst plots, bred, host coll. 3 Jul. 1970, wasp emerged 28 May 1971, ex Streptanus sordidus female (NHMUK010265152); 1 q, England, Bucks, Princes Risborough, Chalk Hills, 30 Jun.-4 Jul. 1943, R.B. Benson leg. (NHMUK010265153); 2 우으, England, Cambs, Chippenham Fen, TL650693, carr/ reedbed, 9-20 Jul. 1984, MT, J. Field leg. (DNPC); 1 , , England, Cambs, Monks Wood, TL202805, Fraxinus/ Ulmus woodland, 26 Jul.-15 Aug. 2005, MT, G.R. Broad leg. (NHMUK010265157); 1 + , England, Norfolk, Santon Downham, TL818883, heath with Betula and Pinus, 29 Jun. 1984, MT, J. Field leg. (DNPC); 1 \&, same collecting data, 1-15 Aug. 1984 (DNPC); 1 q, England, Surrey, Horsley, 11 Jul. 1937, G.E.J. Nixon leg. (NHMUK010265154); 1 \%, England, Surrey, Thames Ditton, 27 Jun. 1970, Z. Bouček leg. (NHMUK010265156); 1 ㅇ, England, Surrey, Weybridge, 2 Jul. 1935, G.E.J. Nixon leg. (NHMUK010265155); 1 +, Wales, Ceredigion, Gwaun Garthenor, SN638558, pan trap, herb-rich meadow, peatland, 24 Jul. 1987, P. Holmes leg. (DNPC); 1 ㅇ, Wales, Clwyd, Sontley Marsh, SJ339481, Carex riparia swamp, 3 Aug. 1988, P. Holmes leg. (DNPC); 1 o, Wales, Swansea, Pant-y-Sais, SS714941, Molina caerulescens/ Potentilla erecta mire, 13 Jul. 1989, P. Holmes leg. (DNPC); 1 +, Scotland, Highland, Rassal National Nature Reserve, 
NG845543, Aug. 1991, MT, P.W. Brown leg. (DNPC); 16 우, Scotland, Perth and Kinross, Ballinluig, 20 Jul. 1977, J.S. Noyes, L. Rogers and T. Huddleston leg. (NHMUK010265159, NHMUK010265160, NHMUK010265161, NHMUK010265162, NHMUK010265163, NHMUK010265164, NHMUK 010265165, NHMUK010265166, NHMUK010265167, NHMUK010265168, NHMUK010265169, NHMUK010265170, NHMUK010265171, NHMUK010265172, NHMUK010265173, NHMUK 010265174).

\section{Distribution}

Austria (new record), Bulgaria (Petrov 1990), Canada (Masner 1976), Czech Republic (Hellén 1964), Denmark (Kolyada \& Chemyreva 2016), Finland (Hellén 1964), France (Kieffer 1916), Germany (Kieffer 1916; Greiler et al. 1992), Italy (Bin et al. 1995; Masner 1976), Kazakhstan (Kolyada \& Chemyreva 2016), Kyrgyzstan (Kolyada \& Chemyreva 2016), Netherlands (Peeters 2015), Norway (Strand 1898), Republic of Ireland (Nixon 1957; Stelfox 1966; O'Connor et al. 2004), Russia (European, Urals) (Kolyada \& Chemyreva 2016), Slovakia (Kolyada \& Chemyreva 2016), Sweden (Kieffer 1916; Nixon 1957), Ukraine (Kolyada \& Chemyreva 2016), United Kingdom (Nixon 1957), USA (Masner 1976).

\section{Host}

Reared from Anteon pubicorne (Dalman, 1818) (Waloff 1975, as Anteon lucidum (Haliday, 1828); Perkins 1976, as Chelogynus lucidus (Haliday, 1828)) and Lonchodryinus ruficornis (Dalman, 1818) (Dryinidae) (Waloff 1975, as Preanteon sp.; Olmi 2000), all from the United Kingdom. Moreover, one specimen was labelled as reared from a female of Streptanus sordidus Zetterstedt, 1828 (Cicadellidae) by V.H. Chambers.

\section{Notes}

Ismarus rugulosus is unusual in that almost all specimens are female, and it is possible that it is normally thelytokous. Only one male specimen, from Germany, was seen during this study.

Ismarus similis Kim, Notton \& Lee sp. nov.

urn:lsid:zoobank.org:act:D6A239E6-659A-44B5-9C36-757819D3A276

Fig. 6

\section{Diagnosis}

In the form of the mesopleuron with its continuous zone of sculpture, Ismarus similis sp. nov. is similar to I. flavicornis. The main difference between these two species (females only) is the mesopleural sculpture, antennal colour and length of median longitudinal furrow on T2: mesopleuron with longitudinal wrinkles, antenna uniformly bright yellowish and T2 with median furrow extending to $2 / 3$ of segment in I. flavicornis; mesopleuron with deep punctures or irregular short wrinkles, antenna not uniformly yellowish and T2 with median furrow not exceeding basal half of segment in I. similis sp. nov.

\section{Etymology}

The specific epithet similis is derived from the Latin adjective, meaning similar.

Type material $(5 \circ$ 우)

Holotype

UNITED KINGDOM: +, Beds, Flitwick Moor, on Lonicera, 26 Jun. 1984, V.H. Chambers leg. (NHMUK010265337).

\section{Paratypes}

UNITED KINGDOM: 2 우, Beds, Mauldon Wood (reared ex dryinid larva from Iassus sp. on Quercus, host coll., 27 Jul. 1979, wasp em., 6 Jun. 1980), V.H. Chamber leg. (NHMUK010265338, 
NHMUK010265339); 1 ㅇ, Surrey, Barnes Common, 14 Jun. 2009, J.S. Noyes leg. (NHMUK010265340); 1 , Norfolk, Sutton Fen, on Quercus, 18 Jul. 1978, V.H. Chambers leg. (NHMUK010265341).

\section{Description}

Female (holotype)

HEAD. Head in dorsal view much wider than long (12:7), slightly wider than width of mesosoma (12:10); POL: 6; LOL: 3; OOL: 5 (Fig. 6B); ocelli large, LOL as long as diameter of lateral ocellus; vertex behind ocelli nearly flat in lateral view; eye large and without setae; inner orbits, frons and temple with few sparse setae; above antennal sockets, face and cheek with numerous long setae; antenna shorter than body length $(4: 5)$; scape and pedicel with scattered setae; A3-A15 with dense and short setae; antennal segments in following proportions (length:width): $22: 5.5 ; 10: 4 ; 17: 3 ; 17: 4 ; 14: 4.5 ; 12: 5$; $12: 5.5 ; 11: 5.5 ; 11: 5.5 ; 11: 5.5 ; 11: 5.5 ; 11: 5.5 ; 11: 5.5 ; 11: 5.5 ; 14: 5.5$ (Fig. 6A).

Mesosoma. Pronotum in dorsal view punctate with whitish long setae along the posterior margin; pronotal shoulders angled; lateral pronotum predominantly punctate to punctate-rugose except smooth

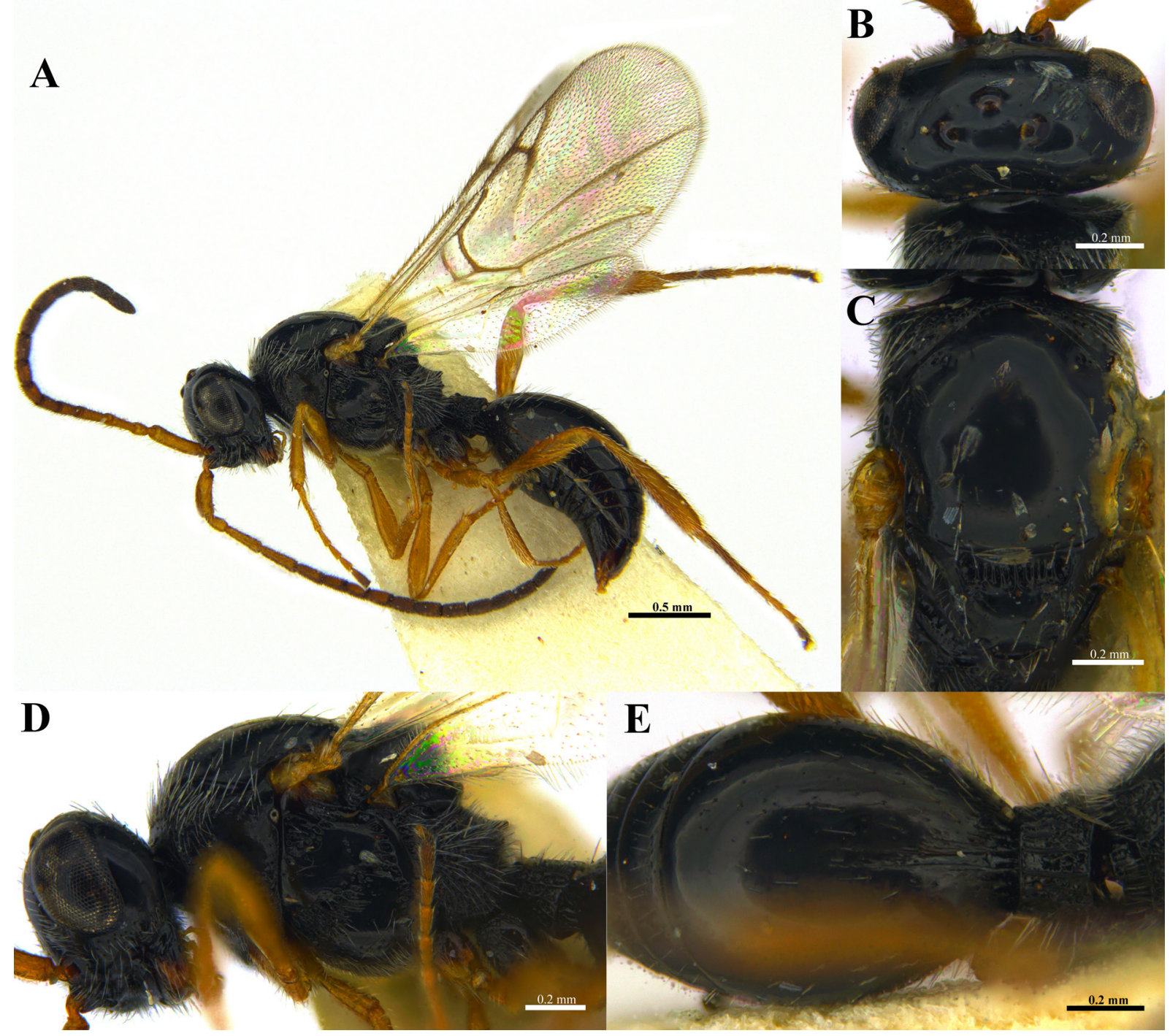

Fig. 6. Ismarus similis Kim, Notton \& Lee sp. nov., holotype, ․ . A. Habitus in lateral view. B. Head in dorsal view. C. Mesosoma in dorsal view. D. Head, mesosoma in lateral view. E. Petiole, T1 in dorsal view. 
and concave in the middle; mesoscutum smooth and convex; notauli present, with 3 small pits anteriorly (Fig. 6C); humeral sulcus finely visible, as long as length of tegula; scutellum smooth and slightly convex, posterior rim rounded (Fig. 6C); anterior scutellar pit large and deep, shorter than remaining scutellar disc, median keel present, strongly crenulate at bottom (Fig. 6C); mesopleuron with a continuous deep punctures to irregular short wrinkles extending from its anteroventral corner up to meso-metapleural suture (Fig. 6D); metapleuron rugose and covered with dense long setae.

WINGs. Fore wing with costal, subcostal, basal, marginal, postmarginal, radial and stigmal veins tubular; medial vein pigmented; radial cell closed, as long as marginal vein and $3.0 \times$ its height (Fig. 6A).

LEGs. Fore and mid legs slender; hind tibiae gradually swollen.

Metasoma. Petiole short and expanded (2: 3), strong costae dorsally (Fig. 6E); tergites completely smooth, with scattered setigerous punctures; base of second tergite with several short costae basally and long and deep median furrow, extending $0.45 \times$ length of second tergite (Fig. $6 \mathrm{E}$ ); sutures between tergites complete and deeply impressed.

Colour. Body black; antennae brown except A1-A4 yellowish brown; legs yellowish brown; legs yellow except basal part of fore and mid coxae dark brown, hind coxae black; tegulae yellow; wings hyaline, covered with brown setae.

Measurements. Head length $0.44 \mathrm{~mm}$, width $0.86 \mathrm{~mm}$; mesosoma length $0.82 \mathrm{~mm}$, width $0.76 \mathrm{~mm}$; metasoma length $1.14 \mathrm{~mm}$; fore wing length $2.00 \mathrm{~mm}$; total body length $2.47 \mathrm{~mm}$.

\section{Variation}

Body length 2.47-3.45 mm; antenna brown except A1-A4 or A1-A8 yellowish brown; median furrow extending $0.40-0.50 \times$ length of second tergite in both sexes.

\section{Host}

Two specimens in NHMUK labelled as reared ex dryinid larva from Iassus sp. (Hemiptera: Cicadellidae: Iassinae) on Quercus sp. An account of the biology is provided by Chambers (1981: as I. halidayi). Probably this species was attacking Anteon infectum (Haliday, 1837) which Chambers reared from the same Iassus.

\section{Distribution}

United Kingdom.

Ismarus spinalis Kolyada \& Chemyreva, 2016

Figs 1F, 2I

Ismarus spinalis Kolyada \& Chemyreva, 2016: 15.

\section{Diagnosis}

Antenna uniformly bright yellowish in female, brown in male; radial cell as long as length of marginal vein in both sexes; A5-A13 subquadrate in both sexes; male A3 and A4 with sharp keels.

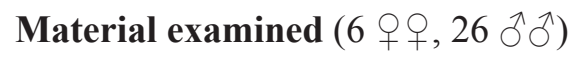

CHINA: 1 đ , Heilongiiang-seong, Shangzhi-shi, 28 Jun. 1999, J.W. Lee leg. (YNU); 1 +, Jilin-seong,

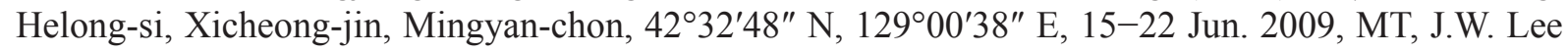
leg. (YNU); 1 §, same collecting data, 25-31 May 2009, MT, J.W. Lee leg. (YNU); 1 \&, same collecting data, 6-22 Jul. 2009, MT, J.W. Lee leg. (YNU). 


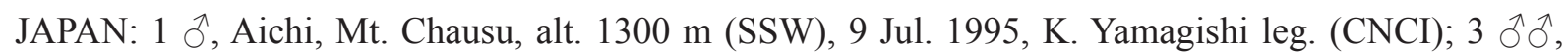
Hokkaido, Sapporo, 18-21 ? 1987, MT, K. Maeto leg. (CNCI); 3 $\lesssim$ , same collecting data, 5 Jun. -2 Jul. 1987, MT, K. Maeto leg. (CNCI); 2 ふ઼ ${ }^{2}$, same collecting data, 18-24 Jun. 1989, MT, K. Maeto leg. (CNCI).

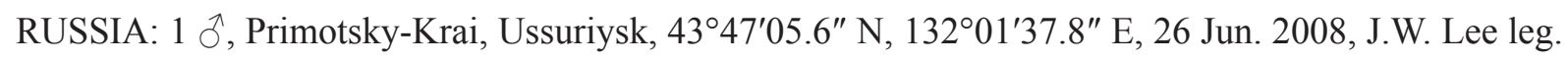
(YNU).

SOUTH KOREA: 1 $\delta^{\lambda}, 1$ q, Chungcheongbuk Province, Boeun-gun, Mt. Songni National Park,

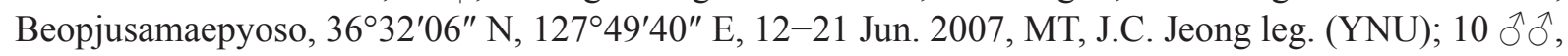
same collecting data, 20 May-3 Jun. 2007, MT, J.C. Jeong leg. (YNU); 1 , Chungcheongnam Province, Gyeryong-si, Sindan-myeon, Bunam-ri, Mt. Gyeryong Donghaksa upper, 14 Mar.-28 Aug. 2007, MT, J.C. Jeong leg. (YNU); 1 ô, Gangwon Province, Donghae-si, Samhwa-dong, Mureung valley, $37^{\circ} 27^{\prime}$ N, $129^{\circ} 1^{\prime}$ E, 2-15 May 2007, MT, J.W. Lee leg. (YNU); 1 , Gangwon Province, Wonju-si, Heungeop-myeon, Maeji-ri, Yonsei University, 21 May-27 Jun. 2014, MT, H.Y. Han leg. (YNU); 1 ð, Gyeongsangbuk Province, Yeongyang-gun, Irwol-myeon, Mt. Irwolsan, 26 Jun.-15 Jul. 2014, MT, H.Y. Han leg. (YNU); 1 , Gyeongsangnam Province, Hamyang-gun, Macheon-myeon, Samjeong-ri, Mt.

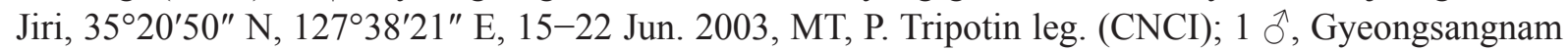
Province, Sanchoeng-gun, Samjang-myeon, Yupyeong-ri, Wangdeungjae 22, Mt. Jiri National Park, $35^{\circ} 23^{\prime} 8.81^{\prime \prime}$ N, 12746'44.11" E, 16 Jun.-20 Sep. 2011, MT, J.W. Lee leg. (YNU).

\section{Variation}

Median furrow extending $0.5-0.8 \times$ length of second tergite in both sexes.

\section{Distribution}

China (Heilongjiang, Jilin) (new record), Japan (Aichi, Hokkaido) (new record), South Korea (new record), Kazakhstan (Kolyada \& Chemyreva 2016), Russia (European, Siberia, Far East) (Kolyada \& Chemyreva 2016).

Ismarus tripotini $\mathrm{Kim} \&$ Lee sp. nov.

urn:1sid:zoobank.org:act:CF814A3F-632E-4BF1-8FF1-9573697B770B

Fig. 7

\section{Diagnosis}

With its small radial cell and darkened trochanter, Ismarus tripotini sp. nov. is similar to I. nigritrochanter Liu, Chen \& Xu, 2011 from the Oriental region, but the main difference between these two is the antennal proportions and posterior scutellar rim shape: antennal flagellomeres are distinctly longer than wide in I. nigritrochanter and slightly longer or quadrate in I. tripotini sp. nov.; posterior scutellar rim rounded in I. nigritrochanter and truncate in I. tripotini sp. nov.

\section{Etymology}

The species is named tripotini after Pierre Tripotin, who collected the specimen which is now the holotype.

\section{Type material $(1$ + $)$}

\section{Holotype}

SOUTH KOREA: ㅇ, Gangwon Province, Chuncheon-si, Nam-myeon, Udong-ri, 26 Jun.-30 Jul. 2006, $\mathrm{MT}$ at forest edge, P. Tripotin leg. (CNCI). 


\section{Description}

Female (holotype)

HeAD. Head in dorsal view much wider than long $(54: 32)$, slightly wider than width of mesosoma (54:44); POL: 11; LOL: 5; OOL: 11 (Fig. 7C); ocelli large, LOL slightly longer than diameter of lateral ocellus $(5: 4)$; vertex behind ocelli nearly flat in lateral view; eye large and without setae; inner orbits, frons and temple with few sparse setae; above antennal sockets, face and cheek with numerous long setae; antenna much shorter than body length $(7: 10)$; scape and pedicel with scattered setae; A3-A15 with dense and short setae; antennal segments in following proportions (length:width): 18:6; 9:5; $12: 4 ; 12: 5 ; 10: 5 ; 9: 6 ; 8: 6 ; 8: 6 ; 8: 6 ; 7: 6 ; 7: 6 ; 7: 6 ; 7: 6 ; 7: 6 ; 12: 6$ (Fig. 7A).

Mesosoma. Pronotum in dorsal view smooth with whitish long setae along the posterior margin; pronotal shoulders angled; upper part of lateral pronotum predominantly smooth and concave in the middle except upper margin with coarsely punctate, lower part of lateral pronotum punctate-rugose; mesoscutum smooth and convex; notauli present anteriorly, oblique long and pit-like, crenulate inside (Fig. 7D); humeral sulcus deep, longer than length of tegula $(3: 2)$; scutellum smooth and slightly convex, posterior rim truncate (Fig. 7D); anterior scutellar pit large and deep, shorter than remaining scutellar disc, median keel present, weakly crenulate at bottom (Fig. 7D); mesopleuron predominantly smooth with deep crenulate line along posterior margin; metapleuron rugose and covered with dense long setae.

WINGS. Fore wing with costal, subcostal, basal, marginal, postmarginal, radial and stigmal veins tubular; medial vein pigmented; radial cell closed, $0.65 \times$ as long as marginal vein and $2.2 \times$ its height (Fig. 7A).

Legs. Fore and mid legs slender; hind tibiae abruptly swollen (Fig. 7B).

Metasoma. Petiole short and expanded (13:17), with irregular longitudinal carinae; tergites completely smooth, with scattered setigerous punctures; base of second tergite with several short costae basally and short and shallow median furrow, extending $0.25 \times$ length of second tergite; sutures between tergites complete and deeply impressed.

Colour. Body black; antennae dark brown except A7-A15 yellowish brown; legs yellow except all coxae and trochanters dark brown; tegulae dark brown; wings hyaline, covered with brown setae.

Measurements. Head length $0.38 \mathrm{~mm}$, width $0.68 \mathrm{~mm}$; mesosoma length $0.91 \mathrm{~mm}$, width $0.55 \mathrm{~mm}$; metasoma length $1.13 \mathrm{~mm}$; fore wing length $2.02 \mathrm{~mm}$; total body length $2.42 \mathrm{~mm}$.

\section{Male}

Unknown.

\section{Host}

Unknown.

\section{Distribution}

South Korea.

\section{Discussion}

Knowledge of the composition of Palaearctic Hymenoptera fauna is essential for the purposes of biological studies requiring accurate identifications, and their applications, including conservation of native species, and the monitoring of faunal change. It is hoped that this paper will be a useful contribution to the ongoing process of documenting Palaearctic Hymenoptera and stimulate further 


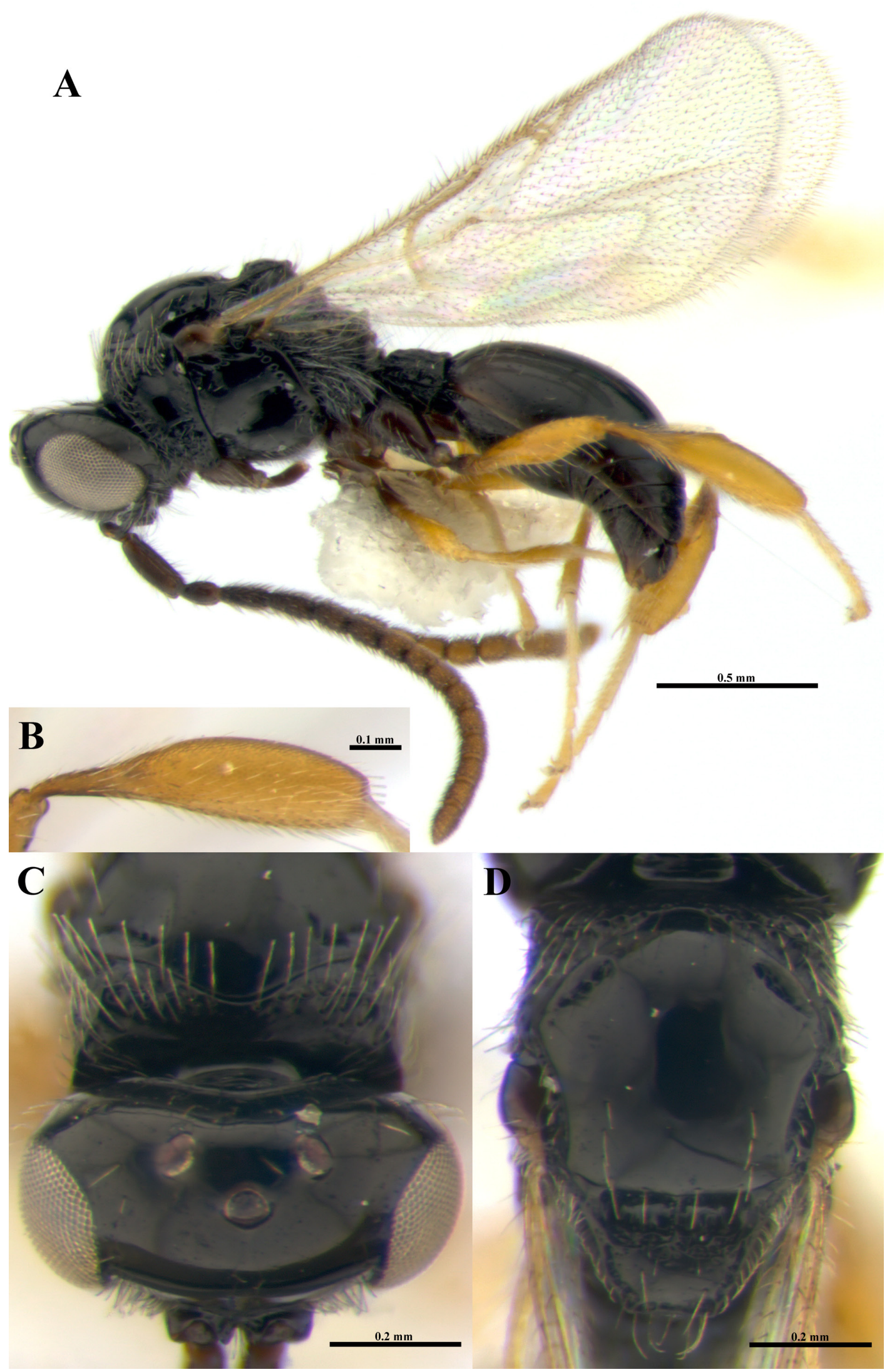

Fig. 7. Ismarus tripotini Kim \& Lee sp. nov., holotype, +. A. Habitus in lateral view. B. Hind tibia. C. Head in dorsal view. D. Mesosoma in dorsal view. 
study of these fascinating creatures. The exploration of new areas in the Palaearctic has uncovered a hitherto unknown diversity among the Ismaridae, viz. five new species, and many new country records, bringing the total to 13 in all, which makes an interesting comparison to the 21 species recorded by Masner (1976) for the New World, and suggests that the Old World diversity may be comparable, or even exceed the New World, when all as yet unstudied areas of the Old World are taken into account.

\section{Acknowledgments}

The authors would like to thank Dr. Lubomir Masner (CNCI), Dr. Zoltán Vas (HNHM), Dr. Hege Vårdal (NHRS) for loaning type or voucher specimens. Hans-Joachim Greiler (Zoologisches Institut I, Universität Karlsruhe), Martin Boness, John S.Noyes (NHMUK), Jeremy Field (University of Sussex), Gavin R. Broad (NHMUK), Paul Eggleton (NHMUK), John W. Ismay (Oxford University Museum of Natural History), Roger Booth (NHMUK), Anthony Galsworthy (NHMUK) and Hyon-Ok Kim (KNA) for donating specimens. Thanks to Arnstein Staverløkk (NINA) for providing additional specimens and taking photos of Ismarus distinctus sp. nov. This work was supported by a grant from the National Institute of Biological Resources (NIBR), funded by the Ministry of Environment (MOE) of the Republic of Korea (NIBR201701203), and the project PROCTONOR as a part of the Taxonomy Initiative of the Norwegian Biodiversity Information Centre.

\section{References}

Alekseev V.N. 1978. A new species of the genus Ismarus Haliday (Hymenoptera, Proctotrupoidea) in the fauna of the USSR. Zoologicheskii Zhurnal 57 (7): 1104-1105.

Bin F., Caleca V., Casale A., Mineo G. \& Pagliano G. 1995. Hymenoptera Proctotrupoidea, Ceraphronoidea. In: Minelli A., Ruffo S. \& La Posta S. (eds) Checklist delle Specie della Fauna Italiana 98: 1-19. Bologna, Calderini.

Chambers V.H. 1955. Some hosts of Anteon spp. (Hym., Dryinidae) and a hyperparasite Ismarus (Hym., Belytidae). The Entomologist's Monthly Magazine 91: 114-115.

Chambers V.H. 1981. A host for Ismarus halidayi Foerst. (Hym., Diapriidae). The Entomologist's Monthly Magazine 117: 29.

Charles J.G. 1989. Edwardsiana crataegi (Douglas) (= Typhlocyba froggatti Baker), Froggatt's apple leafhopper (Homoptera: Cicadellidae). In: Cameron P.J., Hill R.L., Bain J., Thomas W.P. (eds) A review of Biological Control of Invertebrate Pests and Weeds in New Zealand 1874 to 1987: 183-186. Technical Communication CAB International Institute of Biological Control 10, CAB International, Wallingford, UK.

Comério E.F. 2014. Diapriidae e Ismaridae (Hymenoptera, Diaprioidea) de áreas de Mata Atlântica do estado de São Paulo. Master thesis, Universidade Estadual Paulista, Brazil. Mestre em Agronomia (Entomologia Agrícola), Universidade Estadual Paulista - UNESP, Campus De Jaboticabal.

Comério E.F., Perioto N.W. \& Lara R.I.R. 2016. A new species of Ismarus Haliday (Hymenoptera: Ismaridae) from Brazil and a new occurrence record for Ismarus gracilis Masner. EntomoBrasilis 9 (3): 197-201.

Curtis J. 1831. British Entomology: Being Illustrations and Descriptions of the Genera of Insects Found in Great Britain and Ireland: Containing Coloured Figures from Nature of the Most Rare and Beautiful Species, and in Many Instances of the Plants upon which they are Found. 380. Cinetus Dorsiger. Curtis J.H., London. https://doi.org/10.5962/bhl.title.8148

Dumbleton L.J. 1937. Apple leaf-hopper investigations. The New Zealand Journal of Science and Technology 18: 866-877. 
Förster A. 1850. Eine Centurie neuer Hymenopteren. Erste Dekade. Verhandlungen des naturhistorischen Vereines der Preußischen Rheinlande und Westfalens 7: 277-288.

Girolami V. \& Camporese P. 1994. Prima moltiplicazione in Europa di Neodryinus typhlocybae (Ashmead) (Hymenoptera: Dryinidae) su Metcalfa pruinosa (Say) (Homoptera: Flatidae). Atti del XVII Congresso nazionale italiano di Entomologia, Udine, 13-18 giugno 1994: 655-658.

Greiler H.J., Tscharntke T., Ming-Hang V., Gathmann A. \& Wesserling J. 1992. Tierökologische Folgen der Flächenstilllegung, Karlsruhe, Endbericht Ministerium für Ländlichen Raum Ernährung, Landwirtschaft und Forsten Baden-Württemberg, Zoologisches Institut I, Universität Karlsruhe.

Guglielmino A. \& Olmi M. 1997. A host-parasite catalog of world Dryinidae (Hymenoptera: Chrysidoidea). Contributions on Entomology, International 2 (2): 165-298.

Guglielmino A. \& Olmi M. 2006. A host-parasite catalog of world Dryinidae (Hymenoptera: Chrysidoidea): first supplement. Zootaxa 1139: 35-62.

Guglielmino A. \& Olmi M. 2007. A host-parasite catalog of world Dryinidae (Hymenoptera: Chrysidoidea): second supplement. Bollettino di Zoologia agraria e Bachicoltura, Ser. II 39: 121129.

Guglielmino A., Olmi M. \& Christoph B. 2013. An updated host-parasite catalogue of world Dryinidae (Hymenoptera: Chrysidoidea). Zootaxa 3740 (1): 1-113. https://doi.org/10.11646/zootaxa.3740.1.1

Haliday A.H. 1835. Essay on parasitic Hymenoptera. Of the Ichneumones Adsciti. Entomological Magazine 2: 458-468.

Haliday A.H. 1857. Note on a peculiar form of the ovaries observed in a hymenopterous insect, constituting a new genus and species of the family Diapriidae. Natural History Review 4: 166-174.

Hellén W. 1964. Die Ismarinen und Belytinen Finnlands (Hymenoptera: Proctotrupoidea). Fauna Fennica 18: 1-68.

Herrich-Schäffer G.A.W. 1840. Nomenclator entomologicus. Verzeichniss der europäischen Insecten; zur Erleichterung des Tauschverkehrs mit Preisen versehen. Zweites Heft. Coleoptera, Orthoptera, Deratoptera und Hymenoptera. Friedrich Pustet, Regensburg.

International Commission on Zoological Nomenclature (ICZN). 1999. International Code of Zoological Nomenclature. Fourth Edition. International Trust for Zoological Nomenclature, London.

Jervis M.A. 1979. Parasitism of Aphelopus species (Hymenoptera: Dryinidae) by Ismarus dorsiger (Curtis) (Hymenoptera: Diapriidae). Entomologist's Gazette 30: 127-129.

Johnson N.F. 1992. Catalog of World species of Proctotrupoidea, exclusive of Platygastridae (Hymenoptera). Memoirs of the American Entomological Institute 51: 1-825.

Johnson N.F. 2016. Fauna Europaea: Diapriidae. Fauna Europaea version 2.6. Available from http://www.fauna-eu.org [accessed 20 Dec. 2016].

Kieffer J.J. 1908. Proctotrypidæ (suite). Species des Hyménoptères d'Europe et d'Algérie 10(3): 289448.

Kieffer J.J. 1916. Diapriidae. Das Tierreich. Vol. 44. Walter de Gruyter \& Co., Berlin.

Kim C.J. 2016. Taxonomy of the Korean Diapriidae and Ismaridae (Hymenoptera: Diaprioidea): morphological and molecular solutions. $\mathrm{PhD}$ thesis, Yeungnam University, Korea.

Kolyada V.A. \& Chemyreva V.G. 2016. Revision of species of the genus Ismarus Haliday, 1835 (Hymenoptera: Diaprioidea: Ismaridae) of the Russian fauna. Far Eastern Entomologist 318: 1-19. 
Kozlov M.A. 1971. Proctotrupoids (Hymenoptera, Proctotrupoidea) of the USSR. Trudy Vsesoyuznogo Entomologicheskogo Obshchestva 54: 3-67.

Kozlov M.A. 1978. Superfamily Proctotrupoidea. In: Medvedev G.S. (ed.) Determination of Insects of the European Portion of the USSR. Vol. 3, Part 2: 538-664. Nauka, Leningrad.

Liu J., Chen H. \& Xu Z. 2011. Notes on the genus Ismarus Haliday (Hymenoptera, Diapriidae) from China. Zookeys 108: 49-60. https://doi.org/10.3897/zookeys.108.768

Marshall T.A. 1873. A Catalogue of British Hymenoptera; Oxyura. Entomological Society of London, London.

Martínez de Murguía L. 1998. Datos preliminares sobre la presencia de Ismarus dorsiger (Haliday, 1831) (Hymenoptera: Diapriidae) en el País Vasco, primera cita en la Comunidad Autónoma. Munibe (Ciencias Naturales - Natur Zientziak) 50: 109-110.

Masner L. 1957. First preliminary report on the occurrence of genera of the group Proctotrupoidea in Czechoslovakia (Second part -superfamily Proctotrupoidea s. str.). Acta faunistica entomologica Musei Nationalis Pragae 2: 83-107.

Masner L. 1976. A revision of the Ismarinae of the New World (Hymenoptera, Proctotrupoidea, Diapriidae). Canadian Entomologist 108: 1243-1266. https://doi.org/10.4039/Ent1081243-11

Masner L. \& García J.L. 2002. The genera of Diapriinae (Hymenoptera: Diapriidae) in the New World. Bulletin of the American Museum of Natural History 268: 1-138.

Mora-Kepfer F. \& Espinoza A.M. 2009. Parasitism and predation of the planthopper Tagosodes orizicolus (Homoptera: Delphacidae) by a dryinid parasitoid in Costa Rica. Revista de Biologia Tropical 57 (Suppl. 1): 203-211.

Nees von Esenbeck. 1834. Hymenopterorum ichneumonibus affinium monographiae, genera europaea et species illustrantes. Vol. 2. J.G. Cotta, Stuttgart.

Nixon G.E.L. 1957. Hymenoptera, Proctotrupoidea, Diapriidae subfamily Belytinae. Handbooks for the Identification of British Insects 8 (3dii), Royal Entomological Society, St Albans, UK.

Notton D.G. 1996. Diapriid wasps (Hym., Proctotrupoidea) from Abbots Moss, Cheshire. Lancashire \& Cheshire Fauna Society 95: 23-24.

O’Connor J.P., Nash R., Notton D.G. \& Ferguson N.D.M. 2004. A catalogue of the Irish Platygastroidea and Proctotrupoidea (Hymenoptera). Occasional Publication of the Irish Biogeographical Society 7 , Irish Biogeographical Society, Dublin.

Ogloblin A.A. 1925. A new species of Ismarus (fam. Diapriidae. sup. Serphoidea). Časopis Československé společnosti entomologické 22: 50-53.

Olmi M. 1984. A Revision of the Dryinidae (Hymenoptera). Memoirs of the American Entomological Institute 37 (1-2), American Entomological Institute, Gainesville, USA.

Olmi M. 1999a. I Driinidi e la loro utilizzazione in lotta biologica: problemi e prospettive. In: Gervasini E. \& Sala A. (eds) Atti Convegno su "Metcalfa pruinosa: diffusione nel continente europeo e prospettive di controllo biologico", S. Donato Milanese, 21 ottobre 1999: 27-34. Sherwood-Foreste ed Alberi Oggi, 55 (Supplemento).

Olmi M. 1999b. Hymenoptera Dryinidae-Embolemidae. Fauna d'Italia 37. Edizioni Calderini, Bologna.

Olmi M. 2000. Bio-ecologia degli Imenotteri Driinidi e loro impiego in programmi di lotta biologica. In: Lucchi A. (ed.) La Metcalfa negli ecosistemi italiani: 93-117. ARSIA, Firenze. 
Peeters T.M.J. 2015. Tangwespparasieten (Hymenoptera: Ismaridae) in De Kaaistoep. In: Peeters T., van Eck A. \& Cramer T. (eds) Natuurstudie in De Kaaistoep en aangrenzende terreinen in Tilburg. Verslag 2014, 20e onderzoeksjaar: 41-46. TWM Gronden BV, Natuurmuseum Brabant and KNNV-afdeling Tilburg.

Petrov S.D. 1990. Three new species of the subfamily Ismarinae (Hymenoptera, Diapriidae) to the fauna of Bulgaria. Nauchni Trudove Plovdivski Universitet Paisii Khilendarski 28 (6): 89-91.

Perkins J.F. 1976. Hymenoptera Bethyloidea (excluding Chrysididae). Handbooks for the Identification of British Insects 6 (3a), Royal Entomological Society, St Albans, UK.

Sharkey M.J., Carpenter J.M., Vilhelmsen L., Heraty J., Liljeblad J., Dowling A.P.G., Schulmeister S., Murray D., Deans A.R., Ronquist F., Krogmann L. \& Wheeler W.C. 2012. Phylogenetic relationships among superfamilies of Hymenoptera. Cladistics 28 (1): 80-112.

https://doi.org/10.1111/j.1096-0031.2011.00366.x

Strand E. 1898. Enumeratio Hymenopterorum Norvegicorum. Entomologisk Tidskrift 19: 71-112.

Stelfox A.W. 1966. A list of the species of Belytinae (Hym. Proctotrupoidea) so far known from Ireland, with a few records of species taken in Great Britain. Proceedings of the Royal Irish Academy 65B: 101-115.

Swezey O.H. 1928. Present status of certain insect pests under biological control in Hawaii. Journal of Economic Entomology 21: 669-676.

Szabó J.B. 1974. Neue Arten und Gattungen der Diapriiden aus der Mongolei (Hymenoptera, Diapriidae). Annales Historico-Naturales Musei Nationalis Hungarici 66: 353-358.

Thomson C.G. 1858. Sver[i]ges Proctotruper. IV. Tribus Diapriini; Tribus V. Ismarini; Tribus VI. Helorini. Öfversigt af Kongliga Vetenskaps-Akademiens Förhandlingar 185815 (7-8): 359-380.

Tussac H. \& Tussac M. 1991. Récapitulatif d'une collecte de Dryinidae et Diapriidae (Hym. Chrysidoidea et Proctotrypoidea). L'Entomologiste 47 (4): 189-194.

Various contributors. 2018. Hymenoptera Online (HOL) [online]. Available from http:/hol.osu.edu [accessed 21 Nov. 2016].

Ventura D., Algarra A., Ros P., Segude C. \& Pujade J. 1997. Presencia de la subfamilia Ismarinae (Hymenoptera, Proctotrupoidea: Diapriidae) en la Península Ibérica. Boletín de la Asociación española de Entomología 21 (1-2): 105-106.

Wall I. 1967. Die Ismarinae und Belytinae der Schweiz. Entomologische Abhandlungen 35: 123-265.

Waloff N. 1975. The parasitoids of the nymphal and adult stages of leafhoppers Auchenorrhyncha: Homoptera) of acidic grassland. The Transactions of the Royal entomological Society of London 126: 637-686.

Waloff N. \& Jervis M.A. 1987. Communities of parasitoids associated with leafhoppers and planthoppers in Europe. In: MacFadyen A. \& Ford E.D. (eds) Communities of Parasitoids Associated with Leafhoppers and Planthoppers in Europe: 281-402. Advances in Ecological Research 17, London, Academic Press Inc. Ltd.

Williams F.X. 1931. Handbook of the Insects and Other Invertebrates of Hawaiian Sugar Cane Fields. Experiment Station of the Hawaiian Sugar Planters' Association, Honolulu. 
Topic editor: Gavin Broad

Desk editor: Kristiaan Hoedemakers

Printed versions of all papers are also deposited in the libraries of the institutes that are members of the EJT consortium: Muséum national d'Histoire naturelle, Paris, France; Botanic Garden Meise, Belgium; Royal Museum for Central Africa, Tervuren, Belgium; Natural History Museum, London, United Kingdom; Royal Belgian Institute of Natural Sciences, Brussels, Belgium; Natural History Museum of Denmark, Copenhagen, Denmark; Naturalis Biodiversity Center, Leiden, the Netherlands; Museo Nacional de Ciencias Naturales-CSIC, Madrid, Spain; Real Jardín Botánico de Madrid CSIC, Spain. 